\title{
Transcriptome-pathology correlation identifies interplay between TDP-43 and the expression of its kinase CK1E in sporadic ALS
}

\author{
Florian Krach ${ }^{1,2,3} \cdot$ Ranjan Batra $^{1,2} \cdot$ Emily C. Wheeler ${ }^{1} \cdot$ Anthony Q. Vu ${ }^{1} \cdot$ Ruth Wang $^{1} \cdot$ Kasey Hutt $^{1} \cdot$ Stuart J. Rabin ${ }^{4}$. \\ Michael W. Baughn ${ }^{2}$. Ryan T. Libby ${ }^{4}$. Sandra Diaz-Garcia ${ }^{2}$. Jennifer Stauffer ${ }^{2}$. Elaine Pirie ${ }^{1,2}$. Shahram Saberi ${ }^{2}$. \\ Maria Rodriguez ${ }^{2} \cdot$ Assael A. Madrigal $^{1} \cdot$ Zacharias Kohl $^{6} \cdot$ Beate Winner $^{3} \cdot$ Gene W. Yeo $^{1,5,7} \cdot$ John Ravits $^{2}$ (i)
}

Received: 11 March 2018 / Revised: 18 May 2018 / Accepted: 23 May 2018 / Published online: 7 June 2018

(c) Springer-Verlag GmbH Germany, part of Springer Nature 2018

\begin{abstract}
Sporadic amyotrophic lateral sclerosis (sALS) is the most common form of ALS, however, the molecular mechanisms underlying cellular damage and motor neuron degeneration remain elusive. To identify molecular signatures of sALS we performed genome-wide expression profiling in laser capture microdissection-enriched surviving motor neurons (MNs) from lumbar spinal cords of sALS patients with rostral onset and caudal progression. After correcting for immunological background, we discover a highly specific gene expression signature for sALS that is associated with phosphorylated TDP43 (pTDP-43) pathology. Transcriptome-pathology correlation identified casein kinase $1 \varepsilon$ (CSNKIE) mRNA as tightly correlated to levels of pTDP-43 in sALS patients. Enhanced crosslinking and immunoprecipitation in human sALS patientand healthy control-derived frontal cortex, revealed that TDP-43 binds directly to and regulates the expression of CSNK1E mRNA. Additionally, we were able to show that pTDP-43 itself binds RNA. CK1E, the protein product of CSNK1E, in turn interacts with TDP-43 and promotes cytoplasmic accumulation of pTDP-43 in human stem-cell-derived MNs. Pathological TDP-43 phosphorylation is therefore, reciprocally regulated by CK1E activity and TDP-43 RNA binding. Our framework of transcriptome-pathology correlations identifies candidate genes with relevance to novel mechanisms of neurodegeneration.
\end{abstract}

Keywords ALS · Neurodegeneration · Laser capture microdissection · RNA-seq · RNA · Gene-expression · TDP-43 · Casein kinase $\cdot$ Motor neuron $\cdot$ Sporadic disease

Florian Krach and Ranjan Batra have contributed equally to this work.

Electronic supplementary material The online version of this article (https://doi.org/10.1007/s00401-018-1870-7) contains supplementary material, which is available to authorized users.

Gene W. Yeo

geneyeo@ucsd.edu

$\triangle$ John Ravits

jravits@ucsd.edu

1 Department of Cellular and Molecular Medicine, Stem Cell Program and Institute for Genomic Medicine, University of California at San Diego, La Jolla, San Diego, CA, USA

2 Department of Neurosciences, University of California at San Diego, La Jolla, San Diego, USA

3 Department of Stem Cell Biology, University Hospital Erlangen, Friedrich-Alexander Universität Erlangen-Nürnberg (FAU), Erlangen, Germany

\section{Introduction}

Amyotrophic lateral sclerosis (ALS) is a neurological disease characterized by progressive degeneration of upper motor neurons (UMNs) and lower motor neurons (LMNs) progressing over time to fatal respiratory failure usually in

4 Neurogenomics Lab, Benaroya Research Institute, Seattle, WA, USA

5 Department of Physiology, Yong Loo Lin School of Medicine, National University of Singapore, Singapore, Singapore

6 Department of Molecular Neurology, University Hospital Erlangen, Friedrich-Alexander Universität Erlangen-Nürnberg (FAU), Erlangen, Germany

7 Molecular Engineering Laboratory, A*STAR, Singapore, Singapore 
three to 5 years $[8,26]$. While about ten percent occur in a Mendelian pattern of inheritance (familial ALS, fALS), the majority of ALS cases (90\%) are sporadic (sALS) with no known genetic cause. The etiology of sALS and the mechanisms underlying neurodegeneration remain elusive [47]. At present, there is no cure for ALS, a dearth of effective therapeutic targets, and no broadly used molecular biomarkers to evaluate therapeutic efficacy. Furthermore, there are currently no available animal models for experimentation in the pathophysiology of sALS. However, several lines of evidence implicate a central role for perturbed RNA homeostasis in both fALS and sALS [29]. (1) Neuronal inclusions containing hyperphosphorylated Trans-Activation Response DNA Binding Protein 43kd (TDP-43) are the most common neuropathological hallmark present in both sporadic and familial ALS $[4,35]$. TDP-43 is an RNA-binding protein (RBP) responsible for multiple RNA-processing functions including regulation of RNA stability, splicing, and translation [27, 37]. (2) A significant number of fALS causing mutations occur in genes encoding RBPs including TARDBP (TDP-43), FUS (TLS), TAF15, HNRNPA2B1, HNRNPA1 and EWSR1 [42]. (3) The most common genetic cause of ALS is the recently discovered hexanucleotide repeat expansion in the gene C9orf72 (C9ALS), where one of the main mechanisms may involve inhibition of RBPs by transcribed toxic RNA and patients exhibit widespread RNA processing alterations [38]. Together, these observations suggest that altered RBP localization and activity are conserved features of motor neuron disease in both fALS and sALS.

Unbiased profiling of expression and RNA processing patterns can reveal disease mechanisms, clinically relevant biomarkers, and converging pathways that underlie heterogeneous phenotypes of the diverse groups of ALS. While the lack of animal models for the sporadic form of the disease has shifted the focus of research to monogenic models, an alternative strategy is to interrogate molecular changes occurring in human patient tissues [14]. Prior studies have primarily used two approaches to study postmortem ALS tissues: homogenized bulk tissue and cell type specificenriched tissues. Homogenized tissues fail to capture the complex heterogeneity of the nervous system in which mainly one neuronal population undergoes degeneration. Meanwhile, cell-enriched profiling selectively examines neuronal RNA pools by dissection [15] or laser capture microdissection (LCM) [12, 13, 22, 39]. Simultaneous profiling of LCM-captured MNs and the immediately surrounding anterior horn tissue have identified robust gene expression differences in the $\mathrm{MN}$ compartment that failed to be identified by analyses of the anterior horn, highlighting the lack of appropriate resolution in studies using homogenized bulk tissues [39]. A comprehensive systems-based gene-expression analysis of $C 9$ orf 72 fALS [12] and a study correlating gene-expression with p62-pathology, a second neuropathological marker for sALS beside TDP-43, has been assessed to further explore disease mechanisms [13]. To date, genome-wide gene expression studies of sALS have not successfully identified useful candidates that explain disease pathology and mechanisms.

Clinical observations suggest that neurodegeneration in ALS begins focally and progresses neuroanatomically over the course of disease. The site of onset and distribution of involvement between UMNs and LMNs are key determinants of phenotype [41] since MNs in anatomical regions distant from the site of onset, while in the path of degradation, often appear to be relatively unaffected or in early stages of degeneration at the time of death [40]. For example, impaired neuronal control of respiratory muscles often occurs early in the clinical course of ALS while other somatic motor functions remain intact [45], and conversely, patients kept alive by maintaining respiratory function on ventilators gradually progress to a locked-in state of total motor control loss [34]. Therefore, sALS is unique in that the degeneration of MNs innervating the respiratory muscles is the critical determinant of survival, whereas other neurodegenerative diseases patients exhibit respiratory distress in more advanced stages of neurodegeneration. Thus, while neuropathology of most degenerative diseases typically progresses uniformly to end-stage neuropathology, in ALS degeneration is dispersed three-dimensionally [40] and staging of ALS neuropathology is currently based on the anatomical distribution of pTDP-43 inclusions, not chronological progression [7].

Consideration of the neuroanatomical progression of ALS can be exploited to carefully dissect and capture molecular signatures of susceptible but surviving neuronal populations [39]. Here we have isolated surviving MNs from the lumbar spinal region of sALS patients with rostral onset and caudal disease progression and relatively early respiratory failure by LCM and profiled gene expression by RNA-sequencing. We applied a stringent analytical approach to identify unique transcriptomic signatures predictive of sALS histopathology. Comparison of transcriptomic and histopathologic data revealed that mRNA expression levels of the kinase CSNK1E were highly correlated to phospho-TDP43 (pTDP-43) pathology. Enhanced crosslinking and immunoprecipitation combined with high-throughput sequencing (eCLIP) [49] demonstrated that TDP-43 bound to the 3' UTR of CSNK1E RNA, which was upregulated in sALS, and loss of TDP-43 function in vitro led to altered $C S N K 1 E$ mRNA levels. CK1E protein encoded by $C S N K 1 E$ displays protein-protein interaction with TDP-43 and increased expression of CSNK1E resulted in an increase in TDP-43 phosphorylation. Thus, we exploited unique features of sALS neurodegeneration to elucidate interplay between gene expression changes, TDP43 and its phosphorylation directly in affected sALS patient tissue. These observations provide an alternative approach 
to uncover mechanisms underlying sALS pathology, and identify CK1E as a possible therapeutic target modulating TDP-43 phosphorylation.

\section{Methods}

\section{Tissue acquisition and pathological screening}

All nervous systems were acquired by way of an Investigational Review Board and Health Insurance Portability and Accountability Act compliant process. The sALS nervous systems were from patients who had been followed during the clinical course of their illness and met El Escorial criteria for definite ALS. Genetic variants that are known to cause ALS were excluded. MN burden (MNB) was scaled from -2 (largely UMN involvement) to + 2 (largely LMN involvement), whereas 0 is an equal contribution of UMNs and LMNs. Nervous systems selected for profiling for sALS were from patients who had bulbar or arm onset of disease and caudally progressing disease and thus had abundant residual MNs in the lumbar region at the time of death. Control nervous systems were from patients from the hospital's critical care unit when life support was withdrawn. Upon death, an on-call tissue acquisition team performed autopsies immediately. Tissue collections were completed within $6 \mathrm{~h}$, usually within $4 \mathrm{~h}$, of death and the entire motor system was dissected and archived for downstream applications by creating two parallel tissue sets from alternating adjacent regions. For molecular studies, segments were embedded in cutting media, frozen on blocks of dry ice and stored at $-70 \mathrm{C}$. For structural studies, the adjacent segments were fixed in $70 \%$ neutral buffered formalin, embedded in paraffin (formalin-fixed paraffin-embedded or FFPE) and stored at room temperature. Because of received spinal radiation therapy, control 42 was withdrawn form study after initial expression profiling.

\section{Laser capture microdissection (LCM) and RNA extraction}

Laser capture microdissection was done according to the protocol described previously [39]. Briefly, 35-50 sections of frozen OCT-embedded human lumbar spinal cord were cut at a thickness of $9 \mu \mathrm{m}$ in a $-18 \mathrm{C}$ cryotome and placed onto uncharged glass slides. The sections were returned immediately to $-78 \mathrm{C}$ after production, and maintained at that temperature for a minimum of $3 \mathrm{~h}$. Staining with cresyl violet acetate was accomplished in a 10-step, timed, nuclease-free immersion process. MNs were microdissected from each slide using a Pixcell IIe Laser Capture Microdissection (LCM) System (Arcturus Bioscience) and CapSureTM Macro LCM Caps (Applied Biosystems). Each LCM session had an upper time limit of $2.5 \mathrm{~h}$. In addition to the $\mathrm{MN}$ enriched LCM material, the remaining anterior horn region was collected to create a second parallel pool. RNA isolation was carried out in each using an RNeasy Micro kit (Qiagen), which employs guanidinium isothiocyanate and 2-mercaptoethanol extraction and column purification, including an on-column DNase I digestion step. Aliquots of each RNA pool were analyzed for quality and quantity on a Bioanalyzer RNA Pico chip (Agilent) and only those samples with RNA Integrity Numbers (RINs) $>5$ or evidence of $28 \mathrm{~S}$ peaks on the electropherogram tracings were advanced to the next step.

\section{RNA-seq library preparation and sequencing}

Total RNA (10 ng) was amplified to cDNA using random priming with the Ovation ${ }^{\circledR}$ RNA-Seq System (NuGEN). This procedure involved initial generation of doublestranded cDNA followed by amplification through production of single-stranded cDNA using the SPIA ${ }^{\circledR}$ process. The single-stranded cDNA was then copied into double-stranded cDNA and quantified using the PicoGreen kit (Invitrogen) assayed with the FUSION system (Packard Biosciences), resulting in a total yield of 3-4 ug amplified cDNA's. Standard concentration curves using bacteriophage lambda DNA were generated for each PicoGreen analysis, and the samples were diluted by tenfold serial dilutions. QC of the amplified cDNA was determined using a Bioanalyzer running an RNA 6000 Nano LabChip (Agilent). Double-stranded cDNA $(1-2 \mu \mathrm{g})$ was fragmented to 150-200 bp sizes using Adaptive Focused Acoustics ${ }^{\mathrm{TM}}$ (Covaris, Inc., Woburn, MA), and QC of the fragmented cDNA was performed using a Bioanalyzer DNA Chip 1000 (Agilent). Fragmented cDNA's were concentrated using the QIAquick PCR Purification Kit (Qiagen) and $200 \mathrm{ng}$ of the fragmented cDNA's (determined following PicoGreen quantitation) were end-repaired, followed by adaptor ligation onto the fragments and amplification using the Encore ${ }^{\circledR}$ NGS Library System I (NuGEN). Library QC was performed using a Bioanalyzer DNA Chip 1000 .

\section{Gene-expression analysis of RNA-seq data using DESeq 2}

Reads were aligned to UCSC genome browser human genome hg19 build using STAR alignment software with standard parameters published previously [24]. The aligned reads were assigned to the gencode annotation (release 17) and RPKMs were calculated to estimate gene expression. Genes with a sum of more than four counts across the samples were considered. Differentially expressed genes were determined by the DESeq 2 software [32] with a $\log _{2}$ fold change $(\mathrm{FC})>|1|$ and an adjusted $P$ value $<0.05$. GO-term 
analysis was performed using the online functional annotation tool DAVID [20] and categories with Benjamini-Hochberg $P$ values $<0.05$ were reported. Hierarchical clustering, $z$-scores and correlations were computed using the seaborn package (version 0.7.1) in python (3.3). To extract genes with a high PC1-eigenvalue, the first 22 principal components were calculated for each gene in the dataset using the $\mathrm{R}$ platform (version 3.3.1). To obtain a dataset with fewer confounding factors, 1000 genes with the highest PC1-eigenvalues were subtracted from the original dataset. Immune and wounding-related genes for testing enrichment/depletion of such were defined as genes that occur in the EMBL-EBI umbrella GO terms 'immune system process' (GO:0002376) and 'response to wounding' (GO:0009611). Hypergeometric test was used to calculate significance. Differentially expressed genes of the reduced dataset were determined as before. The iterative randomized dataset was generated by shuffling and picking 370 genes (RPKM sum across samples $>0$ ) using the pandas program (version 0.18.1) in python. Spearman correlation coefficients were calculated for each random gene set for all samples and averaged across 10,000 iterations and clustered as described before.

\section{Fluidigm qPCR validation}

High-throughput qPCR validation was carried out on Fluidigm's Biomark HD system, as described in manufacturer's protocol. Specifically, cDNA (6.25 ng) was pre-amplified using $50 \mathrm{nM}$ pooled primer mixture and TaqMan PreAmp Master Mix (Applied Biosystems) for 13 cycles. Unincorporated primers were removed with $8 \mathrm{U}$ of Exonuclease I (NEB) and the reaction products were diluted 10-fold in TE Buffer (TEKnova). The treated cDNA's (280 pg) were combined with SsoFast EvaGreen Supermix with Low ROX (Bio-Rad) and loaded onto the 96.96 Dynamic Array integrated fluidic circuit (Fluidigm). The overlap of Fluidigm results with RNA-seq was performed by comparing the foldchanges found for the specific pairwise comparison. For a particular gene in a single pairwise comparison, if Fluidigm fold-changes was greater than a $\log _{2}$ (fold change) $>12 \mid$ in the same direction as indicated by the RNA-seq, the gene was considered to be validated positive.

\section{H\&E and IHC staining and analysis of spinal cord sections}

$\mathrm{H} \& \mathrm{E}$ was performed in the standard manner. For IHC, sections were stained with antibodies raised against CX3CR1 (sc-20432, Santa Cruz Biotech, Inc.), phosphorylated TDP43 (CAC-TIP-PTD-P02, Cosmo Bio) and CSNK1E (A302135A, Bethyl Laboratories, Inc.).
CX3CR1-positive cells in the areas surrounding morphologically intact (viable at the time of autopsy) MNs (100 $\mu \mathrm{m}$ radius) were counted for five MNs per patient and control. For each slide, we quantified the number of total morphologically intact MNs (tMNs) as well as the number of phosphorylated TDP-43 (pTDP-43) positive MNs. Percentage of pTDP-43 positive MNs was calculated by normalizing pTDP-43 positive MNs to tMNs. The percent surviving MNs for each slide was calculated by normalizing sALS tMNs to control tMNs. Two users quantitatively analyzed three lumbar SC sections per sALS patient in a blind manner.

\section{Molecular staging and correlation of gene expression with pTDP-43}

sALS patients were staged as 'mild' or 'severe', depending on their gene expression clustering. 'mild' and 'severe' has no correlation with disease severity or duration of disease. Additionally, patients were grouped into a 'low' and 'high' pTDP-43 group with the estimated percentages of neurons containing pTDP-43 accumulations. The 'low' and 'high' pTDP-43 was defined by the assumption that the highest rank in pTDP-43 status in the 'mild' group defines the cutoff between 'low' and 'high' pTDP-43. The hypothesis was tested using Fisher's exact test.

RPKMs were $\log _{2}$ transformed, filtered $\left(\log _{2}\right.$ RPKM $\left.>1\right)$ and correlated with the respective percentage of pTDP-43 in sALS patients using a Pearson correlation coefficient. A stringent value of $r>10.8 \mid$ was considered as highly correlating. The randomized correlation set was determined by permuting the estimated percentages five times across all samples.

\section{TDP-43 RNA targets in post-mortem brain tissue}

To identify TDP-43 RNA targets, eCLIP was performed and the datasets were processed as previously published [49]. Briefly, the frozen frontal cortex was homogenized in liquid nitrogen and UV-cross linked in PBS. Afterwards, the tissue was frozen again and stored at $-80{ }^{\circ} \mathrm{C}$ for further processing. An equal amount of tissue from the five donors (ctrl, $n=2$; sALS, $n=3$ ) was lysed, sonicated and cleared. TDP-43 was immunoprecipitated on Dynabeads using an anti-TDP-43 antibody (Bethyl Laboratories Inc., A303223A). After transfer on nitrocellulose, a $70 \mathrm{kDa}$ fragment (beginning from $40 \mathrm{kDa}$ ) was cut from the membrane of the IP and the respective size-matched input (SMinput) control. Sequencing libraries were sequenced with 50 bp paired-end sequencing at a depth of $\sim 10$ million reads for each library. For the analysis, reads were adapter trimmed and mapped with STAR (v2.4.0i) to the human genome (hg19). Custom scripts were used to remove PCR duplicated reads as previously described [49]. CLIPper was used to call peaks [31]. 
Peak regions that passed a $\log _{2}$ fold change (reads in IP/ reads in input) greater than 1 and $a-\log _{10} p$ value (determined from a Fisher's exact test) greater than 2 were kept for downstream analyses. Kvector (https://github.com/olgabot/ kvector) was used to count 6-mers in all peaks that passed filtering criteria $\left(-\log _{10} p\right.$ value $>2, \log _{2}$ fold change $>2$ over input). 6-mers were also counted from a random shuffled background list of peaks with the same genic distribution as foreground peaks. The frequency of occurrence for each 6-mer was compared in the foreground and background using a $Z$ test. 6 -mers that are in the top $5 Z$ scores of any dataset were identified as the most highly enriched and are included on the plot in Fig. S7a (Online Resource 12). For the cross-comparison, significant peaks were assigned to genes using the gencode annotation. First, the transcripts of controls and sALS were overlapped within their respective group, generating a set of transcripts consistently bound in either controls or sALS. Subsequent identification of the overlap between the two groups resulted in a gene set significantly bound by TDP-43 in all five samples. This set was considered highly conserved and used for further downstream applications for overlap between TDP-43 binding and pTDP-43 correlating genes. To estimate if the remaining genes, not bound consistently in all samples, are part of a biological effect or rather due to heterogeneity, the sets were sequentially overlapped with each sample (conserved in controls with all sALS samples, and vice versa).

\section{Validation and cross-analysis with previously published datasets}

For validation of TDP-43 eCLIP signal in CSNK1E in the human brain, TDP-43 ENCODE eCLIP-seq dataset (ENCSR584TCR) and a previously published TDP-43 CLIP-seq dataset in mouse brain [37].

\section{IPSC culture}

An overview of all lines is present in Table S4 (Online Resource 17). The CV-B iPSC-line has been described previously [18]. The study approval for Ctrl-1-SC11 was granted by the local ethics committee (No. 4485, FAU Erlangen-Nuernberg, Germany). IPSC were cultured on Matrigel coated dishes and fed all $24 \mathrm{~h}$ with mTesR1 (Stemcell Technologies). Upon reaching 80\% confluency, iPSC were passaged using Accutase in a 1:3-1:6 ratio. After passaging and thawing, mTesR 1 was supplemented with $10 \mu \mathrm{M}$ Y 27632 (ROCK-inhibitor, RI, Tocris).

\section{MN differentiation}

Human iPSC were differentiated according to using dualSMAD inhibition [9] which has been previously modified to generate MNs and characterized by our group [33]. IPSC were plated with $100 \%$ confluency on Matrigel-coated dishes. One day later, differentiation was induced by changing the media to DMEM/F12 + GLUTAMAX (Thermo Fisher Scientific) with 1\% N-2 and 2\% B-27 Supplement (Thermo Fisher Scientific), $100 \mu \mathrm{M}$ Ascorbic acid (SigmaAldrich) and $1 \%$ Pen/Step (media combination further referred as MN-base), supplemented with $1 \mu \mathrm{M}$ Dorsomorphin (DO), $10 \mu \mathrm{M}$ SB431541 (SB) and $4 \mu \mathrm{M}$ CHIR99021 (Tocris) daily until day six of differentiation. From day seven through day 15 , the cells were fed with MN-base supplemented with $1 \mu \mathrm{M}$ DO, $10 \mu \mathrm{M} \mathrm{SB}, 5 \mu \mathrm{M}$ RI, $200 \mathrm{nM}$ Smoothened Agonist (SAG, EMD Biosciences) and $1.5 \mu \mathrm{M}$ Retinoic Acid (RA, Sigma Aldrich). On day 15, the cells were split with the option to freeze the precursors in liquid nitrogen. For further differentiation, the cells were seeded at a density of 55,555 cells per $\mathrm{cm}^{2}$ on Poly-D-Lysine (SigmaAldrich) + Laminin (Thermo Fischer Scientific) coated plates in MN-base supplemented with $200 \mathrm{nM} \mathrm{SAG}, 1.5 \mu \mathrm{M}$ RA, $5 \mu$ M RI, $2 \mathrm{ng} / \mathrm{mL}$ each of Ciliary Neurotrophic Factor (CNTF), Brain-Derived Neurotrophic Factor (BDNF), and Glial Cell-Derived Neurotrophic Factor (GDNF) (Peprotech). For further differentiation into MN, cells were fed for 3 days from day 22 on with MN-base with $2 \mu \mathrm{M}$ DAPT (Tocris), $5 \mu \mathrm{M}$ RI, and $2 \mathrm{ng} / \mathrm{ml}$ BDNF, GDNF and CTNF. The final maturation step is induced on day 25 by withdrawal of DAPT. For this, the cells were washed with MN-base and further cultured in MN-base supplemented with $5 \mu \mathrm{M}$ RI and $2 \mathrm{ng} / \mathrm{ml}$ BDNF, GDNF and CTNF. The cells were harvested on day 30 for biochemical analyses. To induce TDP-43 phosphorylation, MNs were stressed with $100 \mu \mathrm{M}$ ethacrynic acid

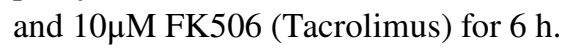

\section{Radiolabeling of RNA bound to protein}

MNs were UV cross-linked and flash frozen. After plus or minus RNase treatment, an antibody specific for pTDP-43 (CAC-TIP-PTD-M01, CosmoBio) or TDP-43 was used to pull down RNA bound to the respective proteins. Wash steps were performed according to the eCLIP-protocol. The RNA was labeled with ${ }^{32} \mathrm{P}$ subsequently. Samples were run on a gel, transferred on a nitrocellulose membrane and developed on a film.

\section{Lentivirus production and transduction}

CSNK1E was cloned from a pENTR221 plasmid (HOC12375, GeneCopoeia) into the pLEX_307 plasmid (gift from David Root, Addgene plasmid \# 41392) using gateway technology. Lentivirus was produced with a secondgeneration virus prep using psPAX2 and pMD2.G plasmids. For knock-down experiments, previously published TARDBP and scrambled (SCR) shRNAs were used [24]. The 
cells were transfected in $293 \times$ T cells in DMEM $+20 \%$ FBS using PEI transfection reagent. The supernatant was collected on day one and day two after transfection and pooled together. Virus was prepared according to the Lenti-X Concentrator kit (Clontech) and concentrated to a 100×. On day 27 and day 28 the respective MN media was supplemented with $1 \%$ of the $100 \times$ virus concentrate and iPSC-derived MN from three genetically different donors were transduced. On day 37, the neurons were fixed in 4\% PFA at RT for 5 min and rinsed in PBS three times. For knock down experiments motor neuron progenitors were infected on day 16 of differentiation and harvested 4 days later. Knock down experiments were performed three independent times in the CV-B cell line.

\section{qPCR}

RNA from motor neuron progenitors was extracted using Trizol and reverse transcribed using SuperScript III (Invitrogen). Power Sybr Green PCR Master Mix (Applied Biosystems) was used to perform qPCR on a BioRad CFX 384 system. $1 \mu \mathrm{M}$ of the following primers were used: CSNK1EF GAATTCCCGTTCTCCTGTGTCTA; CSNK1E-R AAA ACCAGGAATGGAAGATGGAG; CSNK1D-F CGTGCC AAGACTAGCTCAGAAAA; CSNK1D-R CAGACTCTA CTTTCTGGCCGTGA; GAPDH-F ACAGTCCATGCCATC ACTGCC; GAPDH-R GCCTGCTTCACCACCTTCTTG; HPRT-F CCTGGCGTCGTGATTAGTG; HPRT-R TCC CATCTCCTTCATCACATC. The delta-delta-Ct method was used to estimate relative gene expression. GAPDH and HPRT served as controls.

\section{Co-Immunoprecipitation and western blot}

For Co-Immunoprecipitation (Co-IP), the cells were lysed in $500 \mu \mathrm{l}$ RIPA buffer (50 mM Tris-HCl (pH 7.4), 1\% NP-40, $0.5 \%$ Na-deoxycholate, $0.1 \%$ SDS, $150 \mathrm{mM} \mathrm{NaCl}, 2 \mathrm{mM}$ EDTA, $20 \mathrm{mM} \mathrm{NaF}$ ) supplemented with protease inhibitor (Roche), and lysed on ice for $15 \mathrm{~min}$, followed by a sonication and $20 \mathrm{~min}$ centrifugation at $18,000 \mathrm{~g}$ for $20 \mathrm{~min}$ at $4{ }^{\circ} \mathrm{C}$. TDP-43 was immunoprecipitated on Dynabeads overnight with an anti-TDP-43 antibody (Bethyl Laboratories Inc., A303-223A). Beads were washed twice with high salt wash buffer (50 mM Tris- $\mathrm{HCl}$ (pH7.4), $1 \mathrm{M} \mathrm{NaCl}$, $1 \mathrm{mM}$ EDTA, $1 \%$ NP-40, $0.1 \%$ SDS, $0.5 \%$ sodium deoxycholate), followed by two washes of wash buffer $(20 \mathrm{mM}$ Tris- $\mathrm{HCl}$ (pH7.4), $10 \mathrm{mM} \mathrm{MgCl} 2,0.2 \%$ Tween-20). Beads were resuspended in $26 \mu \mathrm{l}$ wash buffer, $10 \mu \mathrm{l} 4 \mathrm{xNuPAGE}$ SDS buffer (Thermo Fisher Scientific) and $100 \mu \mathrm{M}$ DTT up to a volume of $40 \mu$. Thirty microliter were added to a 4-12\% SDS Page gel (Thermo) for CSNK1E western blot, and $10 \mu \mathrm{l}$ were applied for TDP-43 IP-validation. Western blot was performed using 4-12\% BT running gels, (10 well,
$1.5 \mathrm{~mm}$ thickness, life technologies) and transferred on a PVDF membrane. NuPAGE running buffers (life technologies) were used. The membranes were blocked with $5 \%$ milk in TBS-T and incubated with the following primary antibodies: TDP-43 (1:5000, Bethyl Laboratories Inc., A303223A), CSNK1E (1:2500, A302-135A, Bethyl Laboratories, Inc.). For detection, TrueBlot secondary anti-rabbit (1:1000, 18-8816-33, Rockland Immunochemicals) was used.

\section{Immunofluorescence staining of spinal cord sections and cultured cells}

Tissue sections were cut from blocks of formalin-fixed paraffin embedded tissue. $6 \mu \mathrm{m}$-thick tissue sections were deparaffinized through histology grade CitriSolv (two times for 15 min each) and a graded alcohol series (100, 90 and 70\% ethanol for $5 \mathrm{~min}$ each). After a $20 \mathrm{~min}$ permeabilization step in $1 \times$ PBS and $0.2 \%$ Triton-X100, antigen retrieval was performed with a $1 \%$ Tris-based antigen unmasking solution (Vector Laboratories) in a pressure cooker at $120{ }^{\circ} \mathrm{C}$ for $20 \mathrm{~min}$. Sections were further blocked with $2 \%$ Fetal Bovine Serum (vol/vol, S11150, Atlanta Biologicals) and incubated with pTDP43 antibodies (1:1000; CAC-TIP-PTD-M01, CosmoBio), CSNK1E (1:500; A302-135A, Bethyl Laboratories, Inc.) and $\beta$ III-tubulin (1:1000; ab107216, Abcam) overnight at $4{ }^{\circ} \mathrm{C}$. Afterwards, sections were washed three times in PBS $1 \mathrm{X}$ and blocked with 2\% Normal Donkey Serum (Millipore S30-100 ml) before incubation with secondary antibodies in PBS, 2\% Normal Donkey Serum for $1 \mathrm{~h}$ at room temperature. For detection of primary antibodies, donkey anti-rabbit, anti-mouse Alexa-488, Cy3 conjugated antibodies (Jackson ImmunoResearch) and goat anti-chicken Alexa 633 conjugated antibody (A-21103, Invitrogen) were used at a 1:500 dilution. An incubation with DAPI $(1 \mu \mathrm{g} / \mathrm{ml})$ followed of a PBS wash was performed. To reduce auto fluorescence noise, quenching with $0.1 \%$ Sudan Black in $70 \%$ EtOH for $15 \mathrm{~s}$ was applied prior to coverslip mounting with ProLong Gold anti-fade mounting media (Invitrogen).

Imaging was performed on a Zeiss LSM 780 confocal microscope at $63 \mathrm{x}$ magnification under the same microscope conditions. Z-stacks of $0.5 \mu \mathrm{m}$ size were taken.

For the staining of in vitro-derived MNs, the fixed cells were permeabilized in $0.3 \%$ Triton-X-100 and blocked with $5 \%$ goat serum in PBS for 30 min, followed by an incubation of the primary antibodies pTDP43 antibodies (1:1000; CAC-TIP-PTD-M01, CosmoBio), CSNK1E (1:1000; A302135A, Bethyl Laboratories, Inc.) and $\beta$ III-tubulin (1:1000; ab107216, Abcam)) at $4{ }^{\circ} \mathrm{C}$ over night. After rinsing the cells twice in PBS, the secondary antibodies were incubated at a 1:500 dilution for $1 \mathrm{~h}$ at room temperature in the dark, subsequently stained with DAPI and rinsed five times in PBS. 
Images were taken with a Zeiss LSM 780 confocal microscope under the same conditions. Images were taken with z-stacks of $1 \mu \mathrm{m}$. The files were analyzed using ImageJ. Briefly, the cell and nucleus were surrounded manually and the pTDP-43 channel is subject to an intensity thresholding. The binary image is now subject for analysis of the accumulations. An accumulation is called if a spot is bigger than $0.1 \mu \mathrm{m}^{2}$. To obtain the number of cytoplasmic accumulations, the number of accumulations called in the nucleus is subtracted from the number of accumulations in the whole cells.

\section{Data accession}

The NCBI GEO accession number for the RNA-seq from post-mortem tissue reported in this paper is GSE76220. The TDP-43 eCLIP-seq dataset from frontal cortex is accessible through GSE103225.

\section{Statistical analysis}

GraphPad Prism 5.0 software was used to compute $P$ values and linear regression analysis of CSNK1E. Pair-wise comparisons were performed using either $t$ test or Mann-Whitney-U-test and significance in contingency tables were determined by Fisher's exact test. A difference was considered as significant if the $P$ value was below 0.05 .

\section{Results}

\section{Laser capture microdissection results in specific enrichment for motor neuron signature}

Laser-capture microdissection (LCM) of lumbar spinal cord sections from 13 sALS patients and 9 control patients (Table 1) was performed to obtain transcriptome-wide gene expression measurements in adult MNs. The sALS patients studied had rostral onset and caudal disease spread and relatively early respiratory failure. Thus, abundant

Table 1 Clinical data of patient and controls and respective RNA integrity number (RIN)

\begin{tabular}{|c|c|c|c|c|c|c|c|c|}
\hline Patient No. & Primary diagnosis & Age & Gender & Site of onset & $\begin{array}{l}\text { Disease } \\
\text { course } \\
\text { (years) }\end{array}$ & Cause of death & PMI $(h)$ & RIN \\
\hline 16 & sALS & 61 & Male & Arm & 2.5 & NA & 3.5 & 6.1 \\
\hline 21 & sALS & 84 & Male & Respiratory \& hand & 1.2 & NA & 2 & 6.8 \\
\hline 27 & sALS & 74 & Male & Bulbar & 3.25 & NA & 4 & 5.2 \\
\hline 34 & sALS & 81 & Female & Bulbar & 1 & NA & 3.5 & 4.3 \\
\hline 48 & sALS & 67 & Male & Bulbar & 1.75 & NA & 6 & 6.8 \\
\hline 60 & sALS & 58 & Female & Bulbar & 3 & NA & 3 & 5.6 \\
\hline 62 & sALS & 52 & Male & Arm & 1.67 & NA & 6 & 5.1 \\
\hline 63 & sALS & 68 & Male & Arm & 2.5 & NA & 5 & 6.4 \\
\hline 79 & sALS & 55 & Male & Arm & NA & NA & 5 & 6.1 \\
\hline 82 & sALS & 54 & Male & Bulbar & 2.5 & NA & 8 & 5.4 \\
\hline 84 & sALS & 56 & Female & Bulbar & 2 & NA & 4 & 5.9 \\
\hline 85 & sALS & 77 & Female & Trunk & 2.3 & NA & NA & 6 \\
\hline 89 & sALS & 36 & Male & Bulbar & 3 & NA & 5 & 5.9 \\
\hline Mean & & 63 & $9 \mathrm{M}, 4 \mathrm{~F}$ & & 2.2 & & 4.6 & 5.8 \\
\hline 10 & Control & 78 & Male & NA & NA & Sepsis/pulmonary hypertension & 2.5 & 5.0 \\
\hline 39 & Control & 77 & Male & NA & NA & Aortic dissection/MSF & 2 & 6.2 \\
\hline 42 & Control & 61 & Male & NA & NA & Brain tumor & 6 & 5.8 \\
\hline 44 & Control & 80 & Female & NA & NA & Liver failure & 5 & 5.8 \\
\hline 65 & Control & 82 & Male & NA & NA & NA & 4 & 5.4 \\
\hline 67 & Control & 77 & Male & NA & NA & NA & 4 & 5.4 \\
\hline 76 & Control & 68 & Male & NA & NA & Sepsis, ARDS, ARF & 4 & 6.0 \\
\hline 78 & Control & 58 & Female & NA & NA & Vasculitis, cerebral hemorrhage & 3 & 5.4 \\
\hline 88 & Control & 78 & Female & NA & NA & CVA, MSF & 3.8 & 5.8 \\
\hline Mean & & 73 & $6 \mathrm{M}, 3 \mathrm{~F}$ & & & & 3.8 & 5.6 \\
\hline
\end{tabular}

$M S F$ multi system failure, $A R D S$ acute respiratory distress syndrome, $A R F$ acute renal failure, $C V A$ cerebrovascular accident 
residual MNs in the lumbar region were often present at the time of death (Fig. 1a). Total RNA was extracted and amplified using random priming, converted into cDNA, linearly amplified, fragmented and prepared as a sequencing library (Fig. 1a). The quality and quantity of products was evaluated at each step using capillary electrophoresis and reporting RNA integrity numbers (RIN; Table 1). High-quality libraries from samples with RIN values $>5$ and/or had 28 and $18 \mathrm{~s}$ peaks in electropherograms were submitted for high-throughput Illumina sequencing. The 22 RNA-seq libraries were sequenced to an average depth of $\sim 28$ million reads per sample [Table S1 (Online Resource 1)].

To evaluate if the capture and microdissection successfully enriched for MNs, we included RNA-seq data from MNs independently differentiated from induced pluripotent stem cells (iPSCs) as comparison for an early MN signature (Fig. 1b), as well as human embryonic kidney cells (HEK293T) as a non-neuronal comparison. We found that essentially all genes in our neuronal panel were enriched in the LCM-isolated MN samples over the HEK293T cells and exhibit a similar pattern as the iPSC-derived MNs. An astrocyte marker $S B 100$ and microglial markers PTPRC and $C X 3 C R 1$ were also enriched in our MN pool (Fig. 1b). We concluded that the LCM followed by RNA-seq approach was successful in obtaining a MN-enriched population of cells from sALS and control patients, recognizing that other cell

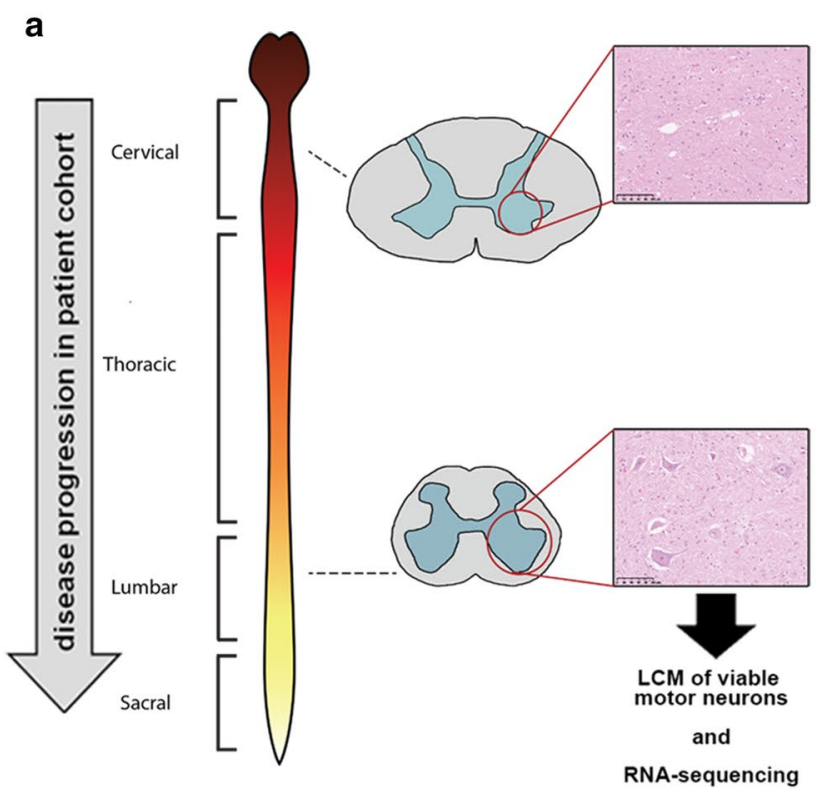

Fig. 1 Laser capture microdissection (LCM) isolates viable lumbar MNs from respiratory onset sALS patients. a Study paradigm: disease progresses caudally in sALS patients with respiratory onset. At the time of death, spinal MNs proximal to the site of onset (cervical) have succumbed, whereas distal MNs (lumbar) remain. These MNs are captured via LCM and subjected to RNA-seq. b Heatmap types such as astrocyte and microglia closely connected with MNs were also unavoidably present.

\section{RNA-seq data analysis of sALS MNs identifies unique gene-expression signature}

We first performed principal component analysis (PCA) on the gene expression data to evaluate if sALS and controls can be distinguished. Intriguingly, most sALS samples and controls tend to cluster separately along the PC1 axis, explaining $49 \%$ of the variance in the data (Fig. 2a). To identify differential expression in LCM-enriched MN samples that distinguish sALS from control individuals, we used DESeq 2 [32], a routinely used software to establish statistically significant gene expression changes. DESeq 2 revealed 972 significantly upregulated genes in the sALS samples relative to controls, with 67 genes with lower expression levels $\left(\log _{2} \mathrm{FC}>1\right.$ and an adj. $P$ value $<0.05$, Data $S 1$ a (Online Resource 2 ). These 1039 genes clearly segregated the control and disease groups (Fig. 2b). Gene ontology (GO)- analysis of the DESeq 2 dataset showed a strong enrichment for genes involved in the immune response and wound healing [Fig. 2c, left side and Table S2 (Online Resource 3)]. To validate the sequencing approach, we additionally used targeted gene-specific high-throughput qRT-PCR analysis on the Fluidigm Biomark platform. Most differentially expressed genes were also found to be dysregulated by qPCR $(78.5 \%)$ [Fig. S1a (Online

b

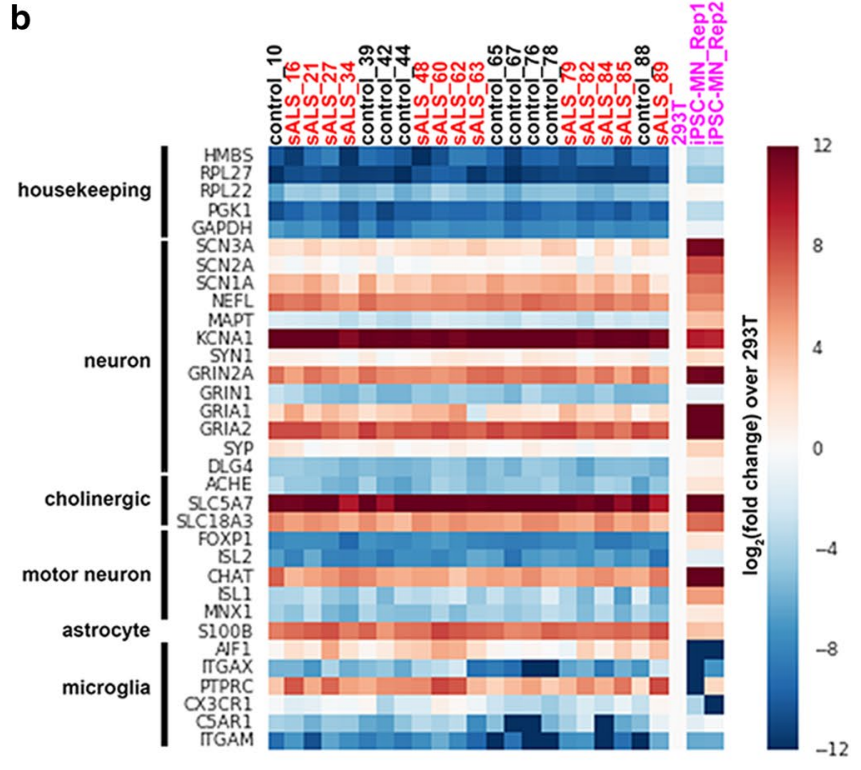

of RNA-seq derived gene expression signature of LCM MNs for controls and sALS samples showing enrichment of neuronal and glial markers. Two independent iPSC-derived MN lines and HEK293T (non neuronal) were used as controls. Color bar indicates $\log _{2}$ fold change over HEK239T non-neuronal cells 
Resource 4)]. Furthermore, the qPCR expression values of 28 tested genes show a tendency to cluster sALS separate from controls [Fig. S1b (Online Resource 4)].

To determine whether this immune signature is intrinsic to neurons or rather a signal from surrounding immune cells, we performed $\mathrm{H} \& \mathrm{E}$ staining of lumbar spinal cord sections from these patients. We detected siderosis [Fig. S2a (Online Resource 5)] and infiltrating potential immune cells around blood vessels [Fig. S2b (Online Resource 5)] to varying degrees in sALS but not in controls. Immunohistochemistry for the microglial marker CX3CR1 (Fig. 2d) showed an enrichment of microglia within a $100 \mu \mathrm{m}$ radius around MNs in sALS (Fig. 2e). In agreement with previous observations [13] and not surprisingly, we observe that there is a substantial presence of the immune cell RNA signature in LCM MN samples in sALS.

\section{Differential gene expression separates sALS patients according to their PTDP-43 status}

Immune activation and wounding-related transcripts were highly differentially expressed in the dataset and most likely mask certain interesting gene expression changes coming from the MN itself. To reduce this signature in an unbiased fashion, we took a closer look at the top 1000 genes contributing to PC1 that accounted for the highest variation and segregated control and sALS samples [Fig. 2a and Data S1b (Online Resource 2)]. These 1000 transcripts show highly enriched immune GO terms (Fig. 2c, right side) and are significantly enriched in immune and wounding-related transcripts (16.6\% of the top 1000 genes, fivefold more than random; $P$ value $=5.11^{*} 10^{-70}$ ). Conversely, when we subtract these 1000 genes from the original dataset, the reduced gene set (whole transcriptome minus $1000 \mathrm{PC} 1^{\text {high }}$ genes) is significantly depleted in immune and wounding transcripts $\left(P\right.$ value $=2.344 * 10^{-68}$; now containing $14 \%$ less immune and wounding related genes). We reanalyzed the reduced RNA-seq data (minus top $1000 \mathrm{PC} 1^{\text {high }}$ genes) with DESeq 2 and observed 370 differentially expressed (DE) genes (330 upregulated and 40 downregulated, $\log _{2} \mathrm{FC}>1$ and an adj. $P$ value $<0.05$, Data S1c [Online Resource 2)]. Encouragingly, this reduced gene set still separated the sALS samples from the controls (Fig. 2f). Intriguingly, we observed six new biological functions (in the top 10 categories) that were not observed in the full dataset or randomly chosen gene sets [Fig. 2g and Table S3 (Online Resource 6)] including 'aging' (FDR adjusted $P$ value $=0.0138$ ) and 'positive regulation of gene-specific transcription' (FDR adjusted $P$ value $=0.0137$ ). Our results indicate that an ALS-specific gene signature independent of immune-related signals can be identified in MNs by LCM.

With these 370 DE genes, a Spearman correlation analysis distinguished the sALS samples into two groups (Fig. 3a, left panel) with patient sample numbers 21, 79 and 63 grouping closer with controls rather than the other sALS samples. Randomly chosen sets of (370) genes did not show clustering of the sALS cases across 10,000 iterations (Fig. 3b, right panel). The gene expression profiles did not cluster differently according to RIN values (RNA quality), post-mortem interval, age, and an obvious involvement of UMN or LMN to the clinical phenotype (Fig. 3a). A clustering similar to the PC-1 adjusted dataset was observed when the genes within the three top gene ontologies ('defense response', 'immune response' and 'response to wounding') were subtracted from the original dataset [Fig S3 (Online Resource 7)].

We next stained sALS patient lumbar spinal cord sections for phosphorylated TDP-43 (pTDP-43), a hallmark of ALS. We examined the control cohort and as expected there was no immunoreactivity against pTDP-43 [Fig S4 (Online Resource 8)]. Expectedly, ALS MNs showed pTDP-43. We ranked the patient cohort in increasing order of pTDP-43 positive MNs [Fig. 3b, c and Fig. S5a (Online Resource 9)]. We observed that samples with a 'mild' RNA signature also had lower pTDP-43 levels, and therefore, Patients 21, 79 and 63 ('mild' RNA signature) are distinct from samples with high pTDP-43 levels $[P$ value $=0.014$ by Fisher's exact test; Figs. 3c and S5b (Online Resource 9)]. Contrarily, samples with 'severe' gene expression changes had higher pTDP-43 pathology. Our results suggest that the 370 gene signature is associated with pTDP-43 pathology in sALS.

\section{CSNK1E expression is linked to TDP-43 and its phosphorylation}

To identify individual genes whose mRNA levels are correlated most with phosphorylated TDP-43 status in the individual sALS patient samples, we calculated genome-wide Pearson correlation coefficients between the neuropathological pTDP-43 status [Data S2a (Online Resource 10)] and $\log _{2}$ RPKM of transcripts [Data S2b (Online Resource 10)] in the patient dataset. We found that the distribution of correlation coefficients resulted in a positively skewed (skewed towards right) distribution. Out of all transcripts detected in the dataset (before subtraction of confounding immune genes), 291 genes were highly correlated $(r>0.8)$ with pTDP-43 pathology, while only 10 genes showed a strong negative correlation in patients $[r<-0.8$, Fig. $4 a$ and Data S2c (Online Resource 10)]. A randomly chosen set of genes illustrated a lack of correlation with pTDP-43 pathology (Fig. 4a, grey curve). In contrast, when correlated with morphologically assessed MN death [Figs. $3 \mathrm{c}$ and S5a (Online Resource 9)], we identified only 11 genes that passed our threshold [ $r>\mid 0.8$, Fig. S6a (Online Resource 11) and Data S2d (Online Resource 10)]. Interestingly, when these pTDP-43-burden correlated genes are compared to a published set of genes correlating with p62-inclusions as a 


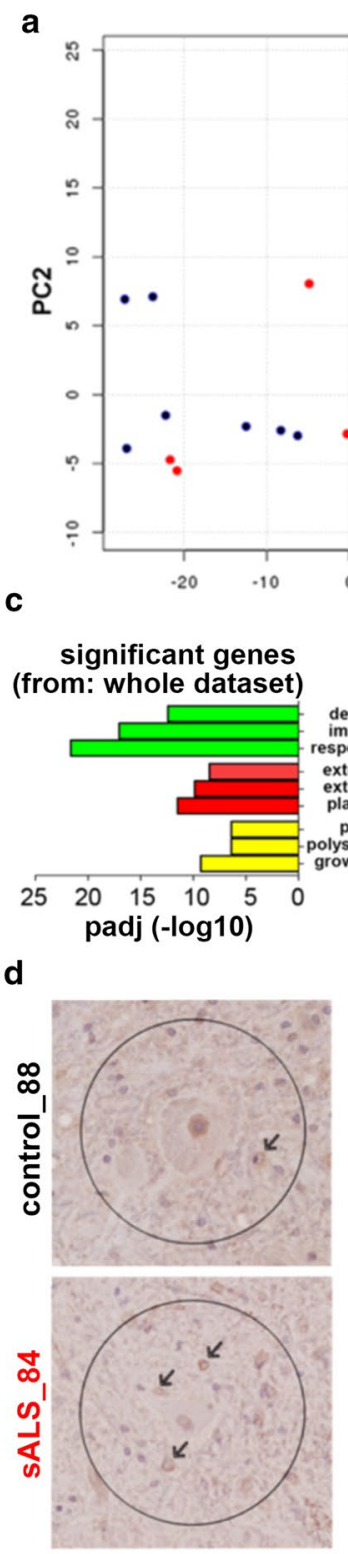

g

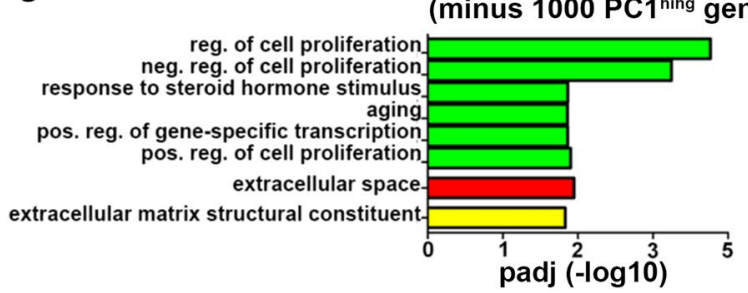

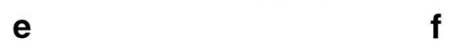

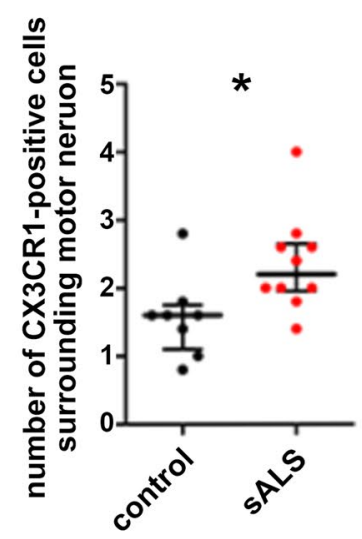
significant genes
(minus $1000 \mathrm{PC1}^{\text {hing }}$ genes) (minus $1000 \mathrm{PC}^{\text {hing }}$ genes)

f b

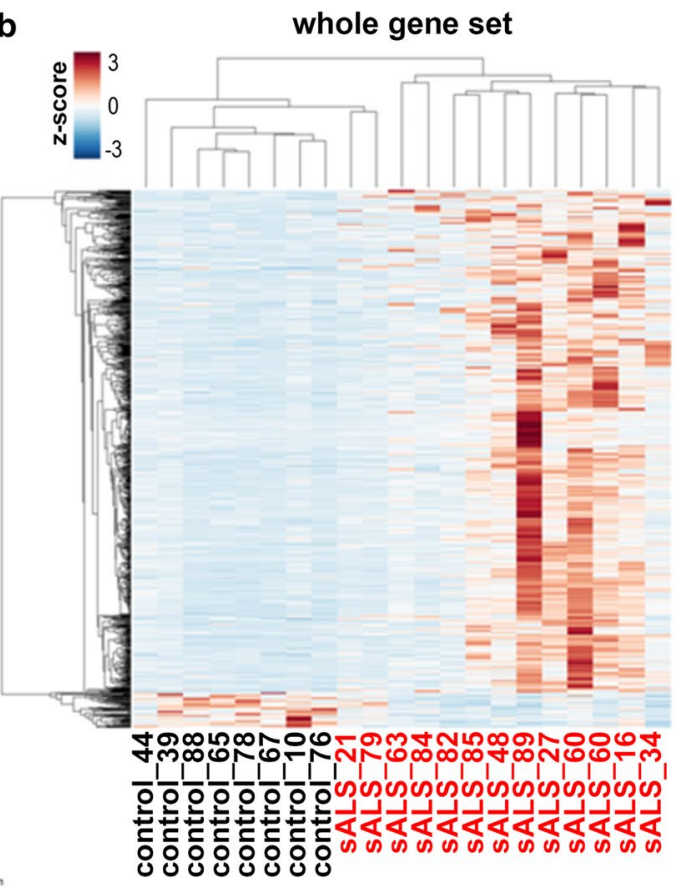

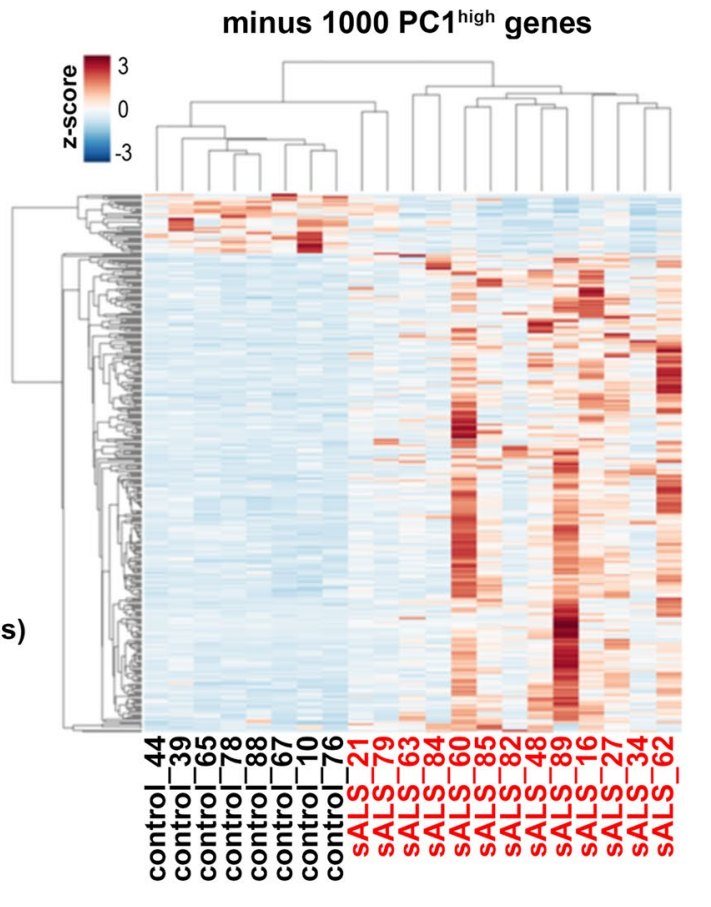

Fig. 2 Differential gene expression analysis in sALS reveals immune signature. a PCA plot representing genome-wide variance of control (blue) and sALS (red) LCM MN sample RNA-seq data along the first two PCs. b Unsupervised hierarchical clustering heatmap by correlation distance of differentially expressed genes determined by DEseq. Color scale represents z-scores. c Three most significant biological function (green), cellular compartment (red) and molecular function (yellow) GO-terms (DAVID) enriched within significantly dysregulated genes (left), and 1000 genes with highest PC1-eigenvalues (right). d Representative immunohistochemistry (IHC) of a control and an SALS patient exhibiting CX3CR1-positive microglia (arrow) within a $100 \mu \mathrm{m}$ radius (circle) of a MN. e Quantification of number of CX3CR1-positive microglia within $100 \mu \mathrm{m}$ radius of $\mathrm{MNs}$, (N sALS $=10, N$ control=9). Graph represents median and interquartile range, Significance was calculated with Mann-Whitney-U-test $(P$ value $=0.0204)$. f Unsupervised correlation-distance based hierarchical clustering heatmap illustrating 370 differentially expressed genes (post filtering out PC1). Color scale represents z-scores. $\mathrm{g}$ Highly significant GO-terms of three GO categories that were not previously reported. Green $=$ biological function, red $=$ cellular compartment, yellow $=$ molecular function 
different neuropathological measure of ALS [13], we only observed an overlap of two genes, raising the possibility of different mechanisms leading to the histopathological hallmarks [Fig S6b (Online Resource 11)].

Next, we asked which of the transcripts that were highly correlated with pTDP-43 burden are also directly bound by the RBP TDP-43. We used enhanced UV cross-linking and immunoprecipitation (eCLIP) in the frontal cortex of two healthy controls and three sALS patients to profile the transcriptome-wide RNA targets bound specific RBPs. eCLIP requires a high cell count, therefore motor cortices which have a higher density of surviving neurons in ALS cases were chosen over spinal cord. The technique features a sizematched input control, enabling the ranked identification of RNA binding sites and RNA substrates above background. Motif analysis of RNA binding sites of TDP-43 confirmed the known GU-rich TDP-43 binding motif in all samples [Fig. S7a (Online Resource 12)]. We found 351 genes $\left(\log _{2}\right.$ FC $>1, P$ value $\left.<0.01\right)$ that show conserved TDP-43 binding in both control and sALS groups [Fig. 4b, Venn diagram and Data S2e (Online Resource 10)]. To estimate if there is any overt tissue-wide loss of TDP-43 RNA binding activity in sALS, we estimated the transcripts bound only by controls [Fig. 4b, blue part of Venn diagram and Data S2f and S2g (Online Resource 10)] or sALS [Fig. 4b, red part of Venn diagram and Data S1h, S2i and S2j (Online Resource 10)] that represent a true loss of TDP-43 binding (and not biological heterogeneity) by analyzing each biological replicate in the control group vs. each biological replicate in the sALS group (pair-wise analysis). Interestingly, only 12 transcripts that were uniquely bound by TDP-43 in controls showed a loss of binding in sALS samples suggesting that analysis of specific cellular populations and not whole brain regions is more likely to uncover any loss of the RNAbinding capability of TDP-43 in sALS (Fig. 4b, pie charts) given the tissue heterogeneity of the frontal cortex.

By comparing the list of genes whose mRNA levels correlated with pTDP-43 burden and genes whose mRNA is bound by TDP-43 in the human brain (Fig. 4c) we identified several interesting candidates, such as the cytoskeleton component $A C T N 1$, the putative splicing factor $L U C 7 L$ and neurodevelopment and regeneration linked gene $N A V I$. Intriguingly, the top candidate was CSNK1E, a kinase that is implicated in TDP-43 phosphorylation [11, 23]. Indeed, regression analysis of pTDP-43 status in the respective patient lumbar spinal cord alone explained more than $93 \%$ of the variance in CSNK1E expression levels in sALS (Fig. 4d). Interestingly, CSNK1E did not fulfill our $\log _{2}>1.0$ fold change stringency criteria of in the standard DESeq 2 analysis in the reduced RNA dataset $\left[\log _{2} \mathrm{FC}=0.9987\right.$, adj. $P$ value $=0.0109$, Data S1c (Online Resource 2)], emphasizing variation in human samples and importance of alternative approaches to average differential expression analyses to capture differences influenced by criteria other than a simple disease status.

\section{TDP-43 directly interacts with the 3 ' UTR of CSNK1E mRNA in human brain and regulates its expression}

To identify the region of the mRNA where TDP-43 binds, we evaluated our frontal cortex eCLIP datasets. We found that TDP-43 directly interacts with the $3^{\prime}$ untranslated region (3' UTR) of CSNK1E in all five brain samples [Fig. 4e and Fig. S7b (Online Resource 12)]. A very strong binding in the 3' UTR of CSNK1E is also observed in ENCODE (K562 cell line) eCLIP datasets [49] [Fig. S7b (Online Resource 12)] and in a previously published mouse brain dataset [37] [Fig. S7c (Online Resource 12)]. To further assess the possibility of RNA-binding capabilities of phospho-TDP-43, we artificially induced TDP-43 phosphorylation in iPSCderived MNs using a previously published combination of ethacrynic acid and tacrolimus [Fig. S8 (Online Resource 13)] [28]. After UV-cross-linking, RNase digestion, and radiolabeling RNA with P-32 we were only able to pull down RNA in treated (induction of TDP-43 phosphorylation) but not non-treated samples using a pTDP-43 specific antibody, suggesting that the phosphorylated TDP-43 indeed binds RNA (Fig. 4f). Combined, our findings are consistent with previous eCLIP data in revealing that TDP-43 binds to the 3'UTR of CSNK1E and suggest a functional role of TDP-43, and potentially even pTDP-43 in regulating CSNK1E mRNA levels.

To elucidate if TDP-43 regulates CSNK1E mRNA levels, we performed TDP-43 knock-down in motor neuron progenitors using an shRNA. CSNK1E mRNA levels were reduced by $40 \%$ ( $P$ value $=0.0271$; Fig. $5 \mathrm{a}$ ), and CK1E protein levels were similarly reduced (Fig. 5c) by $40 \%$ with TDP-43 knock down. Interestingly, the mRNA levels of the CSNK1E homologue CSNK1D remained unchanged (Fig. 5b).

\section{CK1E promotes TDP-43 phosphorylation in human motor neurons}

The Drosophila CK1E homologue known as doubletime is known to phosphorylate mutant TDP-43 [11]. We therefore tested if CK1E-positive MNs show pTDP-43 aggregation. Immunofluorescence of lumbar spinal cord sections indicated that pTDP-43 positive MNs from sALS patients also showed CK1E staining (Fig. 6a), but no co-localization between CK1E and pTDP-43 [Figs. 6a, S9 (Online Resource 

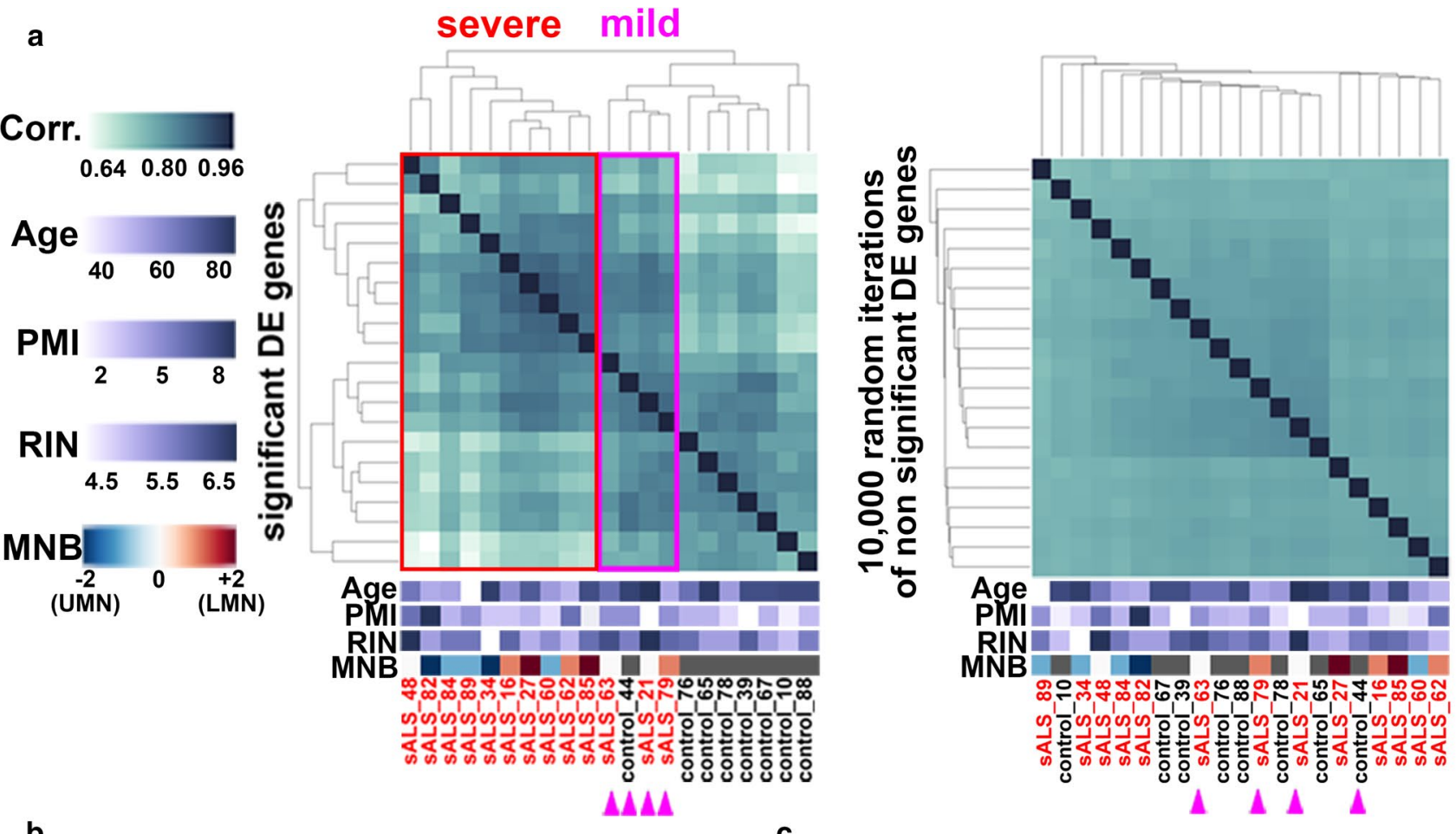
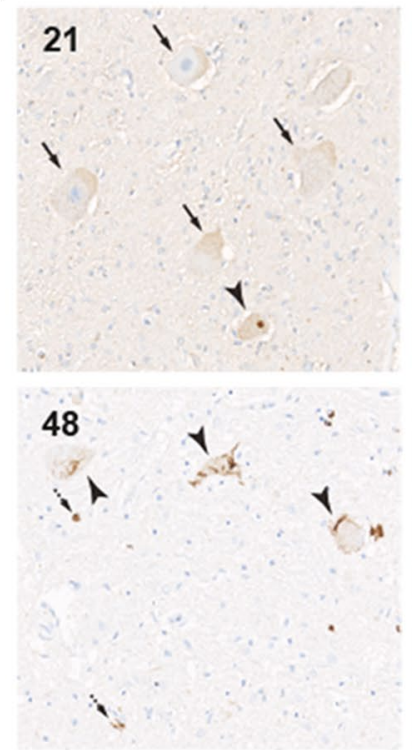

79
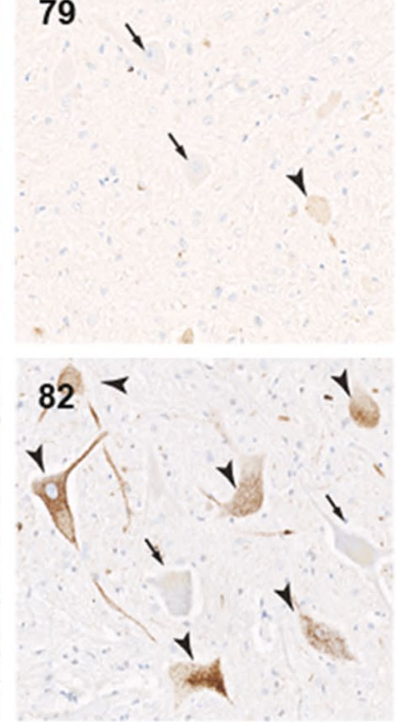

c

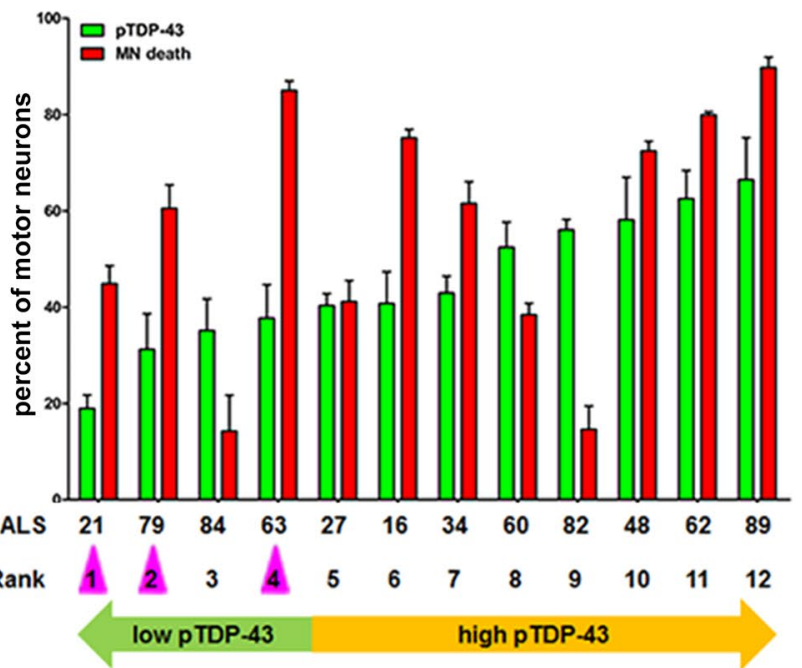

Fig. 3 Unsupervised clustering stages sALS patients in two groups based of pTDP-43 status. a Correlation heatmap clustering sALS (red) and control (black) LCM MN samples according to 370 differentially expressed genes (left) or 10,000 $\times 370$ random genes (right). Color scale represents correlation coefficients. Magenta arrowheads mark samples in the 'mild' gene expression group. Age, postmortem interval (PMI) and RNA integrity number (RIN) for individuals are represented in purple color scales. Motor neuron burden (MNB) of sALS patients indicates primarily upper MN (UMN) involvement $(-2)$ in blue scales, lower MN (LMN) burden $(+2)$ in red scales and

equal contribution (0) of both MNs in white. b Representative IHC of pTDP-43 in sALS patients. Arrow marks morphologically normal MNs, dashed arrow marks morphologically degenerated MNs, arrowhead indicates pTDP-43 within MNs. c Quantification of TDP43-positive and MN degeneration in each patient (3 lumbar sections per sALS patient). Samples were ranked by their percentage of pTDP-43-positive MNs. Green arrow indicates 'low' $p$ TDP-43 group, while yellow marks the 'high' group. Magenta arrowheads indicate sALS samples clustering in 'mild' gene expression group 
a

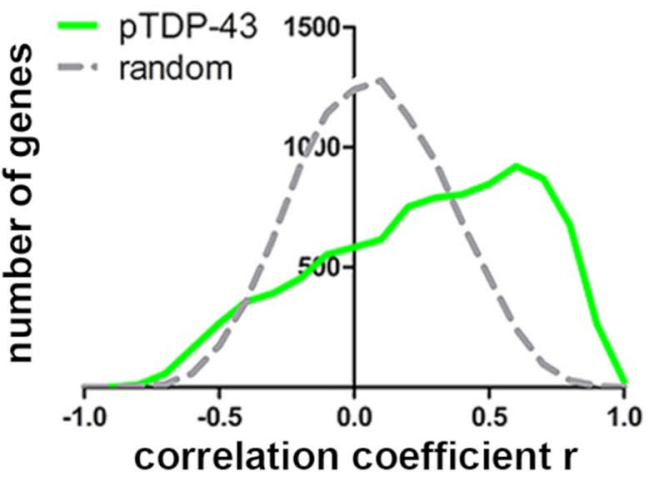

C

$$
r>|0.8|
$$

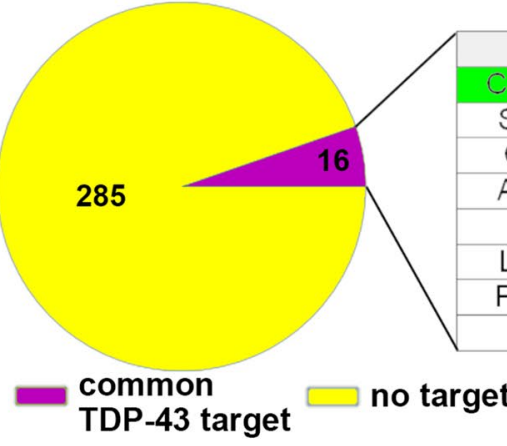

b

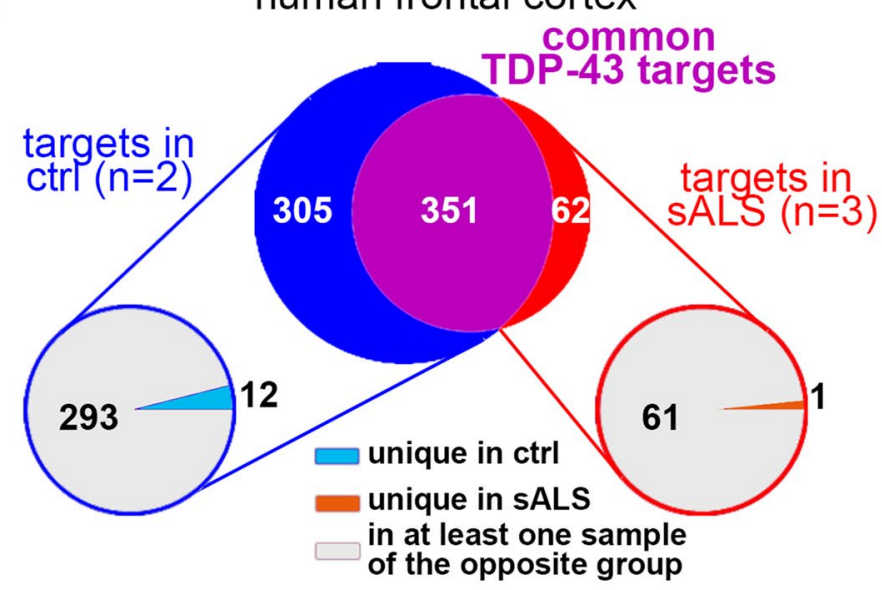

d

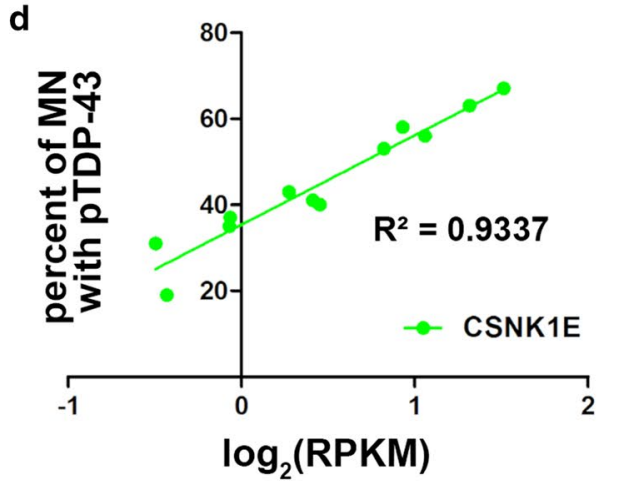

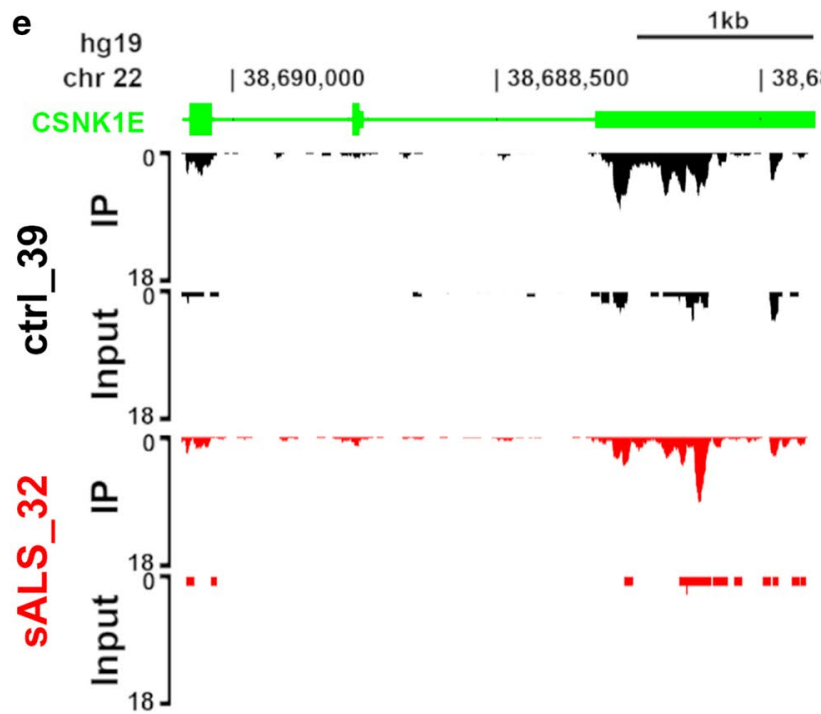

Fig. 4 Neuropathology vs. gene expression correlations and TDP43 eCLIP reveal potential candidate genes. a Histogram of Pearson correlation coefficients $\left(\log _{2}\right.$ RPKM gene expression vs. pTDP-43 metric) for each patient sample (green) or randomized values (grey). Analysis performed with all detected transcripts before subtraction of immune genes. b TDP-43 eCLIP-seq of motor cortex from controls $(n=2)$ and sALS patients $(n=3)$. Venn diagram shows overlapping genes between control $(n=2)$ and sALS $(n=3)$ identified by TDP-43 eCLIP. Blue shows genes identified in both control samples (305), red shows genes identified in all sALS samples (62), magenta shows genes identified in all samples (351). Of the 305 genes conserved between both control samples, 293 were identified in at least one
sALS sample (left pie chart). Of the 62 genes conserved between all sALS samples, 61 were identified in at least one control sample (right pie chart). c Pie chart showing genes highly correlated with TDP-43 pathology ( $r>|0.8|, 301$ genes) with 16 genes that are also bound by TDP-43. CSNK1E shows the highest correlation $(r=0.96)$ and is bound by TDP-43. d Scatter plot illustrating regression of CSNK1E $\log _{2}$ RPKM vs. observed pTDP-43 status in respective sALS patients with fitted line and $R^{2}$ value. e UCSC genome browser track shows binding of TDP-43 in the 3' UTR of CSNK1E in the TDP-43 CLIP but not in the inputs (no IP control). f Radiolabeling of RNA pulled-down with pTDP-43- (left) and TDP-43-specific antibody in unstressed and stressed MNs 
Fig. 5 TDP-43 regulates CSNK1E mRNA levels. a CSNK1E RNA levels determined by qPCR. Graph represents mean + SEM, significance was calculated with $t$ test $(P$ value $=0.0271)$. b CSNK1D RNA levels determined by qPCR. Graph represents mean + SEM, significance was calculated with $t$ test $(P$ value $=0.4538)$. $\mathbf{c}$ Representative western blot showing reduced CK1E protein levels in TDP-43 knock down conditions
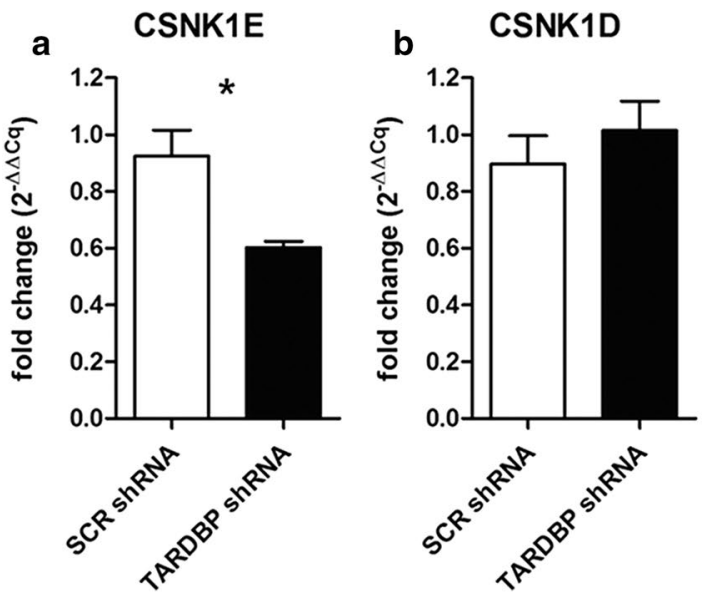

14)], differences in cellular localization or cell type specificity in the whole spinal cord were observed [Fig. S10 (Online Resource 15)]. Next, we performed immunoprecipitation with an antibody against TDP-43, followed by western blot for CK1E to determine whether TDP-43 protein directly associated with CK1E in MNs differentiated from iPSC lines from three healthy individuals and one fALS patient with a TDP-43 N352S mutation. Indeed, we found co-immunoprecipitation of CK1E in all four samples (Fig. 6b). Finally, to determine if CK1E upregulation increases TDP-43 phosphorylation and aggregation, we generated three separate iPSC-derived MN lines (iPSC-MN) from healthy donors stably expressing CSNK1E via lentiviral transduction (Fig. S11 (Online Resource 16)]. Remarkably, CK1E overexpression resulted in significantly more TDP-43 cytoplasmic accumulations compared to mock transfected MNs in each cell line (Fig. 6c, d). Therefore, our results demonstrate that CK1E interacts with and promotes TDP-43 phosphorylation in human MNs.

\section{Discussion}

Neuropathology in neurodegenerative disease is traditionally considered to be a descriptive observation in endstages of the neurodegenerative processes. We exploited the unique anatomical progression of neurodegeneration in ALS patients to capture spared but at-risk MNs in the spinal cord and identify gene-expression signatures of sALS. Our approach utilized the following three criteria: (1) Tissues were collected specifically anticipating genomic studies using short post-mortem intervals and embedding tissues for cyrosectioning. (2) Patients had rostral symptom onset and caudal disease spread leaving residual spinal cord MN populations destined for degeneration in the lumbar region. 3) Optimizing detection of neuronal RNA by laser capturing residual MNs to generate the RNA pools. Indeed, results showed that the pools were highly enriched for mature neuronal markers and we identified several differentially expressed genes.

\section{Robust RNA-seq data analysis detects Immune system involvement in sporadic ALS}

We found more than 1000 differentially expressed genes using DEseq 2 and validated candidate genes using highthroughput qPCR, achieving a concordance rate of $78.5 \%$. Not surprisingly, a strong activation of the immune system including microglia was identified which was confirmed histologically. The microglial marker gene CX3CR1 was highly upregulated. CX3CR1 protein is the juxtacrine receptor for fractalkine localized on the plasma membrane of neurons and is believed to have a disease-modifying role in humans [30] and a protective role in the SOD1 G93A ALS mouse model [26]. We found significant upregulation of markers of adaptive immune cells such as CD4,CD22 and CXCR4 [Data S1a (Online Resource 2)], suggesting infiltration of peripheral immune cells such as T-lymphocytes. T-lymphocytes appear to play a protective role in SOD1 G93A mouse models $[6,10]$. Additionally, a strong enrichment of complement system genes was detected among the upregulated dataset, including $C 1 Q B, C 1 Q C$ and $C 3$ [Data S1a (Online Resource 2)]. Complement system involvement is implicated in neuromuscular junctions [5] and spinal cords of ALS patients [46] before. Interestingly, our data indicates an increase in expression of the F3 tissue factor, the major initiating protease in the blood coagulation cascade. Vascular changes and blood-CNS barrier impairment causing microhemorrhages have been reported in SOD1 ALS previously [16, 17, 43], making this an interesting area for further analysis in the sporadic form of the disease. The presence of components of immune system in the ALS CNS is previously documented [3, 25, 48]. 


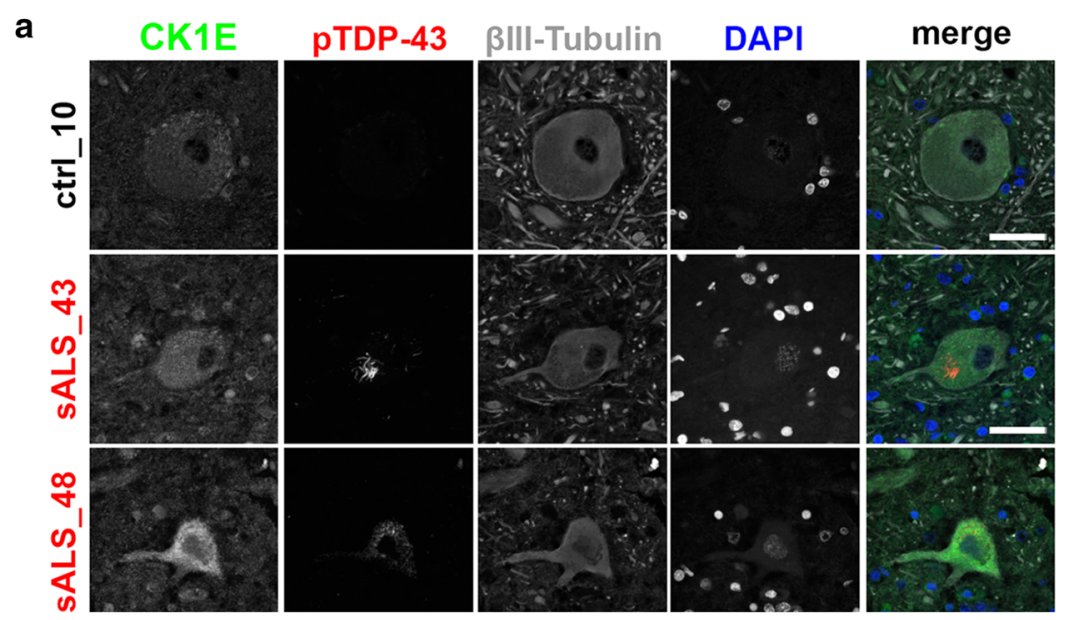

b
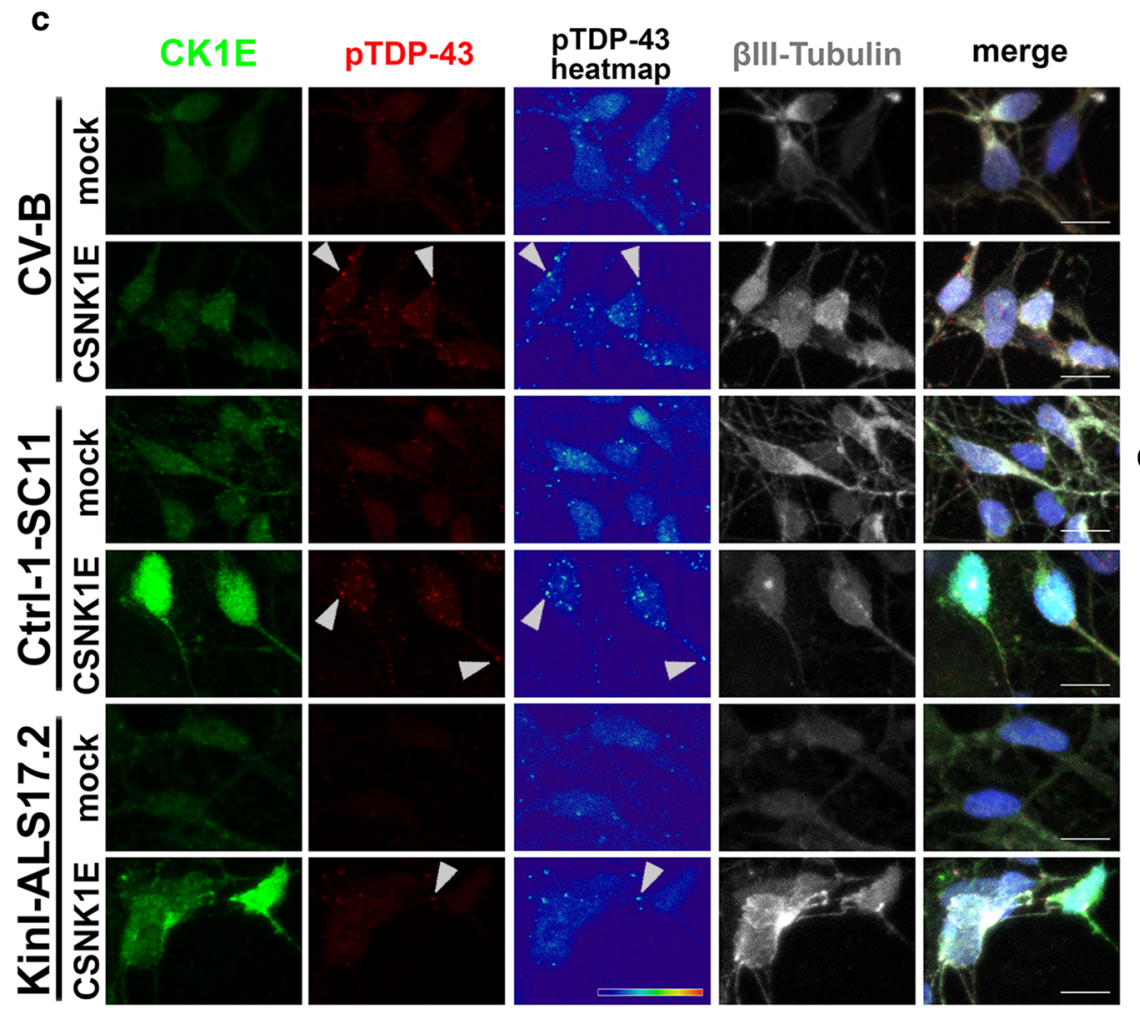

d
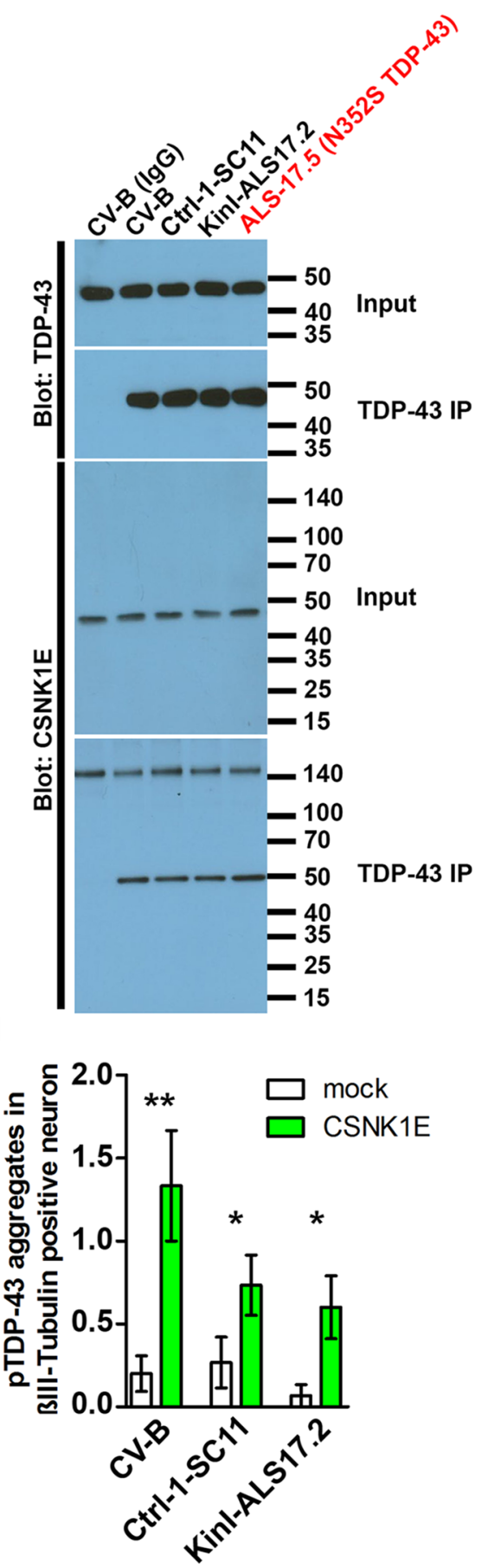

Fig. 6 TDP-43 is a substrate of CK1E. a Immunohistochemistry for CK1E and pTDP-43 in lumbar spinal cord sections show co-occurrence of these signals in the same cell. $\beta$-III tubulin marks neurons. Scale bar $20 \mu \mathrm{m}$. b Immunoprecipitation (IP) of TDP-43 from three control and one fALS patient (TARDBP mutation) iPSC-derived MNs shows CSNK1E co-IP with TDP-43 but not IgG. c Immunofluores-

Identification of immune cells and their receptors that interact with neuronal ligands may help in therapeutic development of neurodegenerative conditions similar to cancer immunotherapy. Our study provides an important

cence (IF) for pTDP-43 after overexpression (OE) of either CSNK1E or mock (control) in three different iPSC-derived MN lines shows increased pTDP-43 aggregates in CSNK1E OE. Scale bar $10 \mu \mathrm{m}$. d Quantification of pTDP-43 signal from CSNK1E or mock OE iPSC$\mathrm{MN}$ lines described in c. Data represented as mean \pm SEM. MannWhitney-U test was used to determine significance

resource of gene-expression signatures of immune cells that may physically interact with MNs.

It is noteworthy that expression level of the ALS-associated frontotemporal-lobar degeneration (FTD) causative 
gene $G R N$ was upregulated before and after filtering datasets. Increased immunoreactivity of progranulin (PGRN), the $G R N$ gene product, is observed in MNs of sALS patients [21]. Since PGRN is also extensively expressed in microglia and upregulated in sALS patients [36], our observation of $G R N$ mRNA levels could be a result of increased number of microglia surrounding MNs. Another possible reason for increased GRN levels could be altered regulation of its mRNA by TDP-43 [37]. Further studies are needed to elucidate the mechanism by which TDP- 43 mediates GRN mRNA stabilization. However, this is consistent with the previous observation of increased GRN mRNA levels in TDP-43 knockdown mouse striatum [37].

These results also invoke the possibility of non-cell autonomous neurotoxicity such that activated microglia or other immune cells directly cause neurodegeneration but such mechanisms need to be further tested. Presence or absence of a cell type in a sample heavily affects differential gene expression profile. Thus, our findings highlight the ability of LCM followed by RNA-seq to enrich, detect and predict pathological cell types in the microenvironment of the targeted cell in an unbiased manner.

\section{Disease characterization based on gene expression signature}

Neurodegenerative disorders lack gene expression-based molecular characterizations especially of the neurons that are primarily affected in the disease and indeed most neurodegenerative disease are far advanced by the time nervous systems are acquired. Despite these issues, we are able to do this in ALS due to the unique anatomical progression, death by respiratory failure, and ready identifications of MNs. Using a set of 370 differentially expressed genes [Data S1d (Online Resource 2)] in a dataset adjusted for confounders as described above, we characterized sALS based on an individual gene expression signature. After filtering immune-related genes, lumbar MNs from rostral onset sALS patients fell into two groups based on individual patient gene expression profiles, and importantly, the group correlating better with control subjects was found to have a significantly lower pTDP-43 burden, consistent with the hypothesis that these MNs were in relatively early stages of degeneration.

Profiling MNs from human spinal cord in this manner is a direct approach to unraveling the complexity of human disease, but clearly drawbacks are that the samples are genetically more variable than animal models and iPSC-derived neuronal cultures. Therefore, we noted two exceptions: control nervous system (\#44) clustered closely with the 'mild' gene expression disease group, and one sALS patient (\#84) that showed low pTDP-43 burden was classified as 'severe'. Since aging was a significant GO term in our dataset, and control 44 is 80 years old (second oldest control individual in our cohort, Table 1), the clustering towards mild sALS might be due to an "aging" signature. Although sALS 84 did not cluster in the mild sALS group, it had higher correlation coefficients compared with the control samples than most of the other patients in the severe group. Clearly, greater sample numbers, stratification by UMN and LMN clinical disease burdens, better age-matched controls, and deeper sequencing in the future will explain such discrepancies that could be due to either false positives, aging, or genetic modifiers. A future independent validation of this gene set in a second patient cohort will solidify the validity of our classifier. More stringent criteria and reproducible measurements that define the two sALS classes (mild and severe gene expression) are required to establish precise high-throughput sequencing based molecular diagnostic marker panels.

\section{Transcriptome-phenotype correlations and TDP-43 eCLIP-seq reveal potential candidate genes}

We aimed to identify candidates that were altered in sALS with increasing pTDP-43 burden using our RNA-seq and TDP-43 eCLIP-seq dataset. Using a similar approach, a gene expression-p62 neuropathology correlation in ALS was recently reported [13]. When we compared the datasets between our (pTDP43 vs. gene-expression) and the aforementioned report (p62 vs. gene-expression), there was an overlap of only two transcripts. This observation may be explained by the fact that in contrast to p62, TDP-43 is an $\mathrm{RBP}$, directly contributing to RNA expression changes in ALS. In addition, there were differences in the spinal cord region that was analyzed. While the current study focuses largely on the lumbar spinal cord in sALS patients who had rostral onset and caudal disease spread, the Cooper-Knock et al. report evaluated cervical spinal cords of lumbar-onset patients with rostral spread. Additionally, Cooper-Knock et al. utilized an equal number of C9orf72 and sporadic ALS patients in their study and a recent publication suggests differential transcriptome expression in the frontal cortex of C9orf72 ALS patients in comparison to sALS patients [38].

\section{Interplay between CSNK1E mRNA levels and TDP-43 phosphorylation}

Using our pTDP-43 correlation analysis combined with TDP-43 eCLIP-seq, we identified interesting candidates such $L U C 7 L, N A V 1$ and ACTN1. Our top candidate using this approach was casein kinase 1 epsilon (CSNK1E). RNA expression levels of CSNK1E exhibited the highest correlation with pTDP-43 status in SALS nervous systems $\left(R^{2}=0.93\right)$ and we also showed for the first time that TDP-43 binds the 3' UTR of CSNK1E mRNA by eCLIP-seq of TDP43 in frontal cortex. Additionally, we showed that TDP-43 loss of function results in reduced CSNK1E levels. It was 
surprising at first that our patient dataset revealed upregulation of CSNK1E mRNA in SALS whereas TDP-43 knockdown showed a decrease in the CSNKIE mRNA levels. However, it appeared plausible considering a recent study of TDP-43 knock-down in mice and an ALS mouse model expressing $\Delta$ NLS-TDP-43 (cytoplasmic overexpression and localization combined with nuclear loss of function) also showed altered gene expression in opposite directions [2]. Combined with our finding that pTDP-43 itself binds RNA, this supports toxic effects of cytoplasmic, pathological and phosphorylated TDP-43 on target RNAs in sALS separate from nuclear loss of function. Therefore, it is likely that disease models that increase cytoplasmic localization and aggregation combined with nuclear loss of TDP-43 function will better represent ALS [50].

It is still a matter of debate whether phosphorylated and aggregated TDP- 43 has protective functions in ALS. The casein kinase family is known to phosphorylate TDP-43 in vitro [19] and several casein kinase 1 subfamily dependent TDP-43 phosphorylation sites have been elucidated [23]. Furthermore, phenotypes and cellular alterations of TDP-43 in GRN mutation linked FTD patient blood derived lymphoblastoid cells can be reversed by two blood-brain barrier permeable CK1D/E inhibitors [1, 44]. Additionally, the fly homologue of CK1E is known to phosphorylate mutant TDP-43 and promotes its aggregation [11]. Our results provide the first evidence for the relevance of CK1E in the human disease since its levels highly correlate with TDP43 phosphorylation status in sALS patients. The evidence that TDP-43 associates with CK1E at the protein level, and ectopic CK1E overexpression in iPSC-derived MNs leads to increased pTDP-43 accumulation, support that CK1E may be a candidate for therapeutic intervention.

In summary, we elucidated individual gene expression profiles in LCM-isolated MN pools using an unbiased methodology and correlated them with pTDP-43 pathology in an effort to characterize early molecular signatures in sALS. Since animal models of sporadic forms of MN diseases do not yet exist, this approach focused on identifying disease features directly in human tissue. Using transcriptome-pathology correlations, we uncover a disease-associated kinase regulating TDP-43 phosphorylation in MNs, and thus identify a candidate for further research and a potential therapeutic target to alleviate $\mathrm{MN}$ degeneration.

Acknowledgements We would like to thank Mark Perelis for helpful comments on the manuscript and the members of the Ravits and Yeo labs for great discussions. G.W.Y. was partially supported by grants from the National Institute of Health (HG004659, NS075449 and U54HG007005), ALS Association and Target ALS. J.R. was supported by grants from the National Institutes of Health (NS051738), Microsoft Research, Target ALS, ALS Association, the Moyer Foundation, the Benaroya Foundation, Pam Golden, the Wyckoff family, and Mrs. Lois Caprile. R.B. is a Myotonic Dystrophy Foundation (MDF) postdoctoral fellow. G.W.Y. is an Alfred P. Sloan Research Fellow. F.K., B.W. and G.W.Y. were supported by the Bavaria California Technology Center (BaCaTeC) (project no. 8 [2015-2]) and B.W. received funding from the German Federal Ministry of Education and Research (BMBF, 01GQ113) and the Bavarian Ministry of Education and Culture, Science, and the Arts in the framework of the Bavarian Research Network Induced Pluripotent Stem Cells ForIPS.

\section{References}

1. Alquezar C, Salado IG, de la Encarnacion A, Perez DI, Moreno F, Gil C, de Munain AL, Martinez A, Martin-Requero A (2016) Targeting TDP-43 phosphorylation by Casein Kinase-1delta inhibitors: a novel strategy for the treatment of frontotemporal dementia. Mol Neurodegener 11:36. https://doi.org/10.1186/s1302 4-016-0102-7

2. Amlie-Wolf A, Ryvkin P, Tong R, Dragomir I, Suh E, Xu Y, Van Deerlin VM, Gregory BD, Kwong LK, Trojanowski JQ, Lee VM, Wang LS, Lee EB (2015) Transcriptomic Changes Due to Cytoplasmic TDP-43 Expression Reveal Dysregulation of Histone Transcripts and Nuclear Chromatin. PLoS ONE 10:e0141836. https://doi.org/10.1371/journal.pone.0141836

3. Anneser JM, Chahli C, Ince PG, Borasio GD, Shaw PJ (2004) Glial proliferation and metabotropic glutamate receptor expression in amyotrophic lateral sclerosis. J Neuropathol Exp Neurol 63:831-840

4. Arai T, Hasegawa M, Akiyama H, Ikeda K, Nonaka T, Mori H, Mann D, Tsuchiya K, Yoshida M, Hashizume Y, Oda T (2006) TDP-43 is a component of ubiquitin-positive tau-negative inclusions in frontotemporal lobar degeneration and amyotrophic lateral sclerosis. Biochem Biophys Res Commun 351:602-611. https ://doi.org/10.1016/j.bbrc.2006.10.093

5. Bahia El Idrissi N, Bosch S, Ramaglia V, Aronica E, Baas F, Troost D (2016) Complement activation at the motor end-plates in amyotrophic lateral sclerosis. J Neuroinflammation 13:72. https ://doi.org/10.1186/s12974-016-0538-2

6. Beers DR, Henkel JS, Zhao W, Wang J, Huang A, Wen S, Liao B, Appel SH (2011) Endogenous regulatory T lymphocytes ameliorate amyotrophic lateral sclerosis in mice and correlate with disease progression in patients with amyotrophic lateral sclerosis. Brain 134:1293-1314. https://doi.org/10.1093/brain/awr074

7. Brettschneider J, Del Tredici K, Toledo JB, Robinson JL, Irwin DJ, Grossman M, Suh E, Van Deerlin VM, Wood EM, Baek Y, Kwong L, Lee EB, Elman L, McCluskey L, Fang L, Feldengut S, Ludolph AC, Lee VM, Braak H, Trojanowski JQ (2013) Stages of pTDP-43 pathology in amyotrophic lateral sclerosis. Ann Neurol 74:20-38. https://doi.org/10.1002/ana.23937

8. Brown RH, Al-Chalabi A (2017) Amyotrophic Lateral Sclerosis. N Engl J Med 377:162-172. https://doi.org/10.1056/NEJMra1603 471

9. Chambers SM, Fasano CA, Papapetrou EP, Tomishima M, Sadelain M, Studer L (2009) Highly efficient neural conversion of human ES and iPS cells by dual inhibition of SMAD signaling. Nat Biotechnol 27:275-280. https://doi.org/10.1038/nbt.1529

10. Chiu IM, Chen A, Zheng Y, Kosaras B, Tsiftsoglou SA, Vartanian TK, Brown RH Jr, Carroll MC (2008) T lymphocytes potentiate endogenous neuroprotective inflammation in a mouse model of ALS. Proc Natl Acad Sci U S A 105:17913-17918. https://doi. org/10.1073/pnas.0804610105

11. Choksi DK, Roy B, Chatterjee S, Yusuff T, Bakhoum MF, Sengupta U, Ambegaokar S, Kayed R, Jackson GR (2014) TDP-43 Phosphorylation by casein kinase Iepsilon promotes 
oligomerization and enhances toxicity in vivo. Hum Mol Genet 23:1025-1035. https://doi.org/10.1093/hmg/ddt498

12. Cooper-Knock J, Bury JJ, Heath PR, Wyles M, Higginbottom A, Gelsthorpe C, Highley JR, Hautbergue G, Rattray M, Kirby J, Shaw PJ (2015) C9ORF72 GGGGCC Expanded Repeats Produce Splicing Dysregulation which Correlates with Disease Severity in Amyotrophic Lateral Sclerosis. PLoS ONE 10:e0127376. https:// doi.org/10.1371/journal.pone.0127376

13. Cooper-Knock J, Green C, Altschuler G, Wei W, Bury JJ, Heath PR, Wyles M, Gelsthorpe C, Highley JR, Lorente-Pons A, Beck T, Doyle K, Otero K, Traynor B, Kirby J, Shaw PJ, Hide W (2017) A data-driven approach links microglia to pathology and prognosis in amyotrophic lateral sclerosis. Acta Neuropathol Commun 5:23. https://doi.org/10.1186/s40478-017-0424-x

14. Cooper-Knock J, Kirby J, Ferraiuolo L, Heath PR, Rattray M, Shaw PJ (2012) Gene expression profiling in human neurodegenerative disease. Nat Rev Neurol 8:518-530. https://doi. org/10.1038/nrneurol.2012.156

15. Dangond F, Hwang D, Camelo S, Pasinelli P, Frosch MP, Stephanopoulos G, Stephanopoulos G, Brown RH Jr, Gullans SR (2004) Molecular signature of late-stage human ALS revealed by expression profiling of postmortem spinal cord gray matter. Physiol Genomics 16:229-239. https://doi.org/10.1152/physiolgenomics .00087 .2001

16. Garbuzova-Davis S, Rodrigues MC, Hernandez-Ontiveros DG, Louis MK, Willing AE, Borlongan CV, Sanberg PR (2011) Amyotrophic lateral sclerosis: a neurovascular disease. Brain Res 1398:113-125. https://doi.org/10.1016/j.brainres.2011.04.049

17. Garbuzova-Davis S, Sanberg PR (2014) Blood-CNS Barrier Impairment in ALS patients versus an animal model. Front Cell Neurosci 8:21. https://doi.org/10.3389/fncel.2014.00021

18. Gore A, Li Z, Fung HL, Young JE, Agarwal S, Antosiewicz-Bourget J, Canto I, Giorgetti A, Israel MA, Kiskinis E, Lee JH, Loh YH, Manos PD, Montserrat N, Panopoulos AD, Ruiz S, Wilbert ML, Yu J, Kirkness EF, Izpisua Belmonte JC, Rossi DJ, Thomson JA, Eggan K, Daley GQ, Goldstein LS, Zhang K (2011) Somatic coding mutations in human induced pluripotent stem cells. Nature 471:63-67. https://doi.org/10.1038/nature09805

19. Hasegawa M, Arai T, Nonaka T, Kametani F, Yoshida M, Hashizume Y, Beach TG, Buratti E, Baralle F, Morita M, Nakano I, Oda T, Tsuchiya K, Akiyama H (2008) Phosphorylated TDP-43 in frontotemporal lobar degeneration and amyotrophic lateral sclerosis. Ann Neurol 64:60-70. https://doi.org/10.1002/ana.21425

20. da Huang W, Sherman BT, Lempicki RA (2009) Systematic and integrative analysis of large gene lists using DAVID bioinformatics resources. Nat Protoc 4:44-57. https://doi.org/10.1038/nprot .2008 .211

21. Irwin D, Lippa CF, Rosso A (2009) Progranulin (PGRN) expression in ALS: an immunohistochemical study. J Neurol Sci 276:913. https://doi.org/10.1016/j.jns.2008.08.024

22. Jiang YM, Yamamoto M, Kobayashi Y, Yoshihara T, Liang Y, Terao S, Takeuchi H, Ishigaki S, Katsuno M, Adachi H, Niwa J, Tanaka F, Doyu M, Yoshida M, Hashizume Y, Sobue G (2005) Gene expression profile of spinal motor neurons in sporadic amyotrophic lateral sclerosis. Ann Neurol 57:236-251. https:// doi.org/10.1002/ana.20379

23. Kametani F, Nonaka T, Suzuki T, Arai T, Dohmae N, Akiyama $\mathrm{H}$, Hasegawa M (2009) Identification of casein kinase-1 phosphorylation sites on TDP-43. Biochem Biophys Res Commun 382:405-409. https://doi.org/10.1016/j.bbrc.2009.03.038

24. Kapeli K, Pratt GA, Vu AQ, Hutt KR, Martinez FJ, Sundararaman B, Batra R, Freese P, Lambert NJ, Huelga SC, Chun SJ, Liang TY, Chang J, Donohue JP, Shiue L, Zhang J, Zhu H, Cambi F, Kasarskis E, Hoon S, Ares M Jr, Burge CB, Ravits J, Rigo F, Yeo GW (2016) Distinct and shared functions of ALS-associated proteins
TDP-43, FUS and TAF15 revealed by multisystem analyses. Nat Commun 7:12143. https://doi.org/10.1038/ncomms12143

25. Kawamata T, Akiyama H, Yamada T, McGeer PL (1992) Immunologic reactions in amyotrophic lateral sclerosis brain and spinal cord tissue. Am J Pathol 140:691-707

26. Kiernan MC (2014) ALS and neuromuscular disease: in search of the Holy Grail. Lancet Neurol 13:13-14. https://doi.org/10.1016/ S1474-4422(13)70226-6

27. Lagier-Tourenne C, Polymenidou M, Hutt KR, Vu AQ, Baughn M, Huelga SC, Clutario KM, Ling SC, Liang TY, Mazur C, Wancewicz E, Kim AS, Watt A, Freier S, Hicks GG, Donohue JP, Shiue L, Bennett CF, Ravits J, Cleveland DW, Yeo GW (2012) Divergent roles of ALS-linked proteins FUS/TLS and TDP-43 intersect in processing long pre-mRNAs. Nat Neurosci 15:1488-1497. https ://doi.org/10.1038/nn.3230

28. Liachko NF, Saxton AD, McMillan PJ, Strovas TJ, Currey HN, Taylor LM, Wheeler JM, Oblak AL, Ghetti B, Montine TJ, Keene CD, Raskind MA, Bird TD, Kraemer BC (2016) The phosphatase calcineurin regulates pathological TDP-43 phosphorylation. Acta Neuropathol 132:545-561. https://doi.org/10.1007/s0040 1-016-1600-y

29. Ling SC, Polymenidou M, Cleveland DW (2013) Converging mechanisms in ALS and FTD: disrupted RNA and protein homeostasis. Neuron 79:416-438. https://doi.org/10.1016/j.neuro n.2013.07.033

30. Lopez-Lopez A, Gamez J, Syriani E, Morales M, Salvado M, Rodriguez MJ, Mahy N, Vidal-Taboada JM (2014) CX3CR1 is a modifying gene of survival and progression in amyotrophic lateral sclerosis. PLoS ONE 9:e96528. https://doi.org/10.1371/journ al.pone. 0096528

31. Lovci MT, Ghanem D, Marr H, Arnold J, Gee S, Parra M, Liang TY, Stark TJ, Gehman LT, Hoon S, Massirer KB, Pratt GA, Black DL, Gray JW, Conboy JG, Yeo GW (2013) Rbfox proteins regulate alternative mRNA splicing through evolutionarily conserved RNA bridges. Nat Struct Mol Biol 20:1434-1442. https://doi. org/10.1038/nsmb.2699

32. Love MI, Huber W, Anders S (2014) Moderated estimation of fold change and dispersion for RNA-seq data with DESeq2. Genome Biol 15:550. https://doi.org/10.1186/s13059-014-0550-8

33. Martinez FJ, Pratt GA, Van Nostrand EL, Batra R, Huelga SC, Kapeli K, Freese P, Chun SJ, Ling K, Gelboin-Burkhart C, Fijany L, Wang HC, Nussbacher JK, Broski SM, Kim HJ, Lardelli R, Sundararaman B, Donohue JP, Javaherian A, Lykke-Andersen J, Finkbeiner S, Bennett CF, Ares M Jr, Burge CB, Taylor JP, Rigo F, Yeo GW (2016) Protein-RNA Networks Regulated by Normal and ALS-Associated Mutant HNRNPA2B1 in the Nervous System. Neuron 92:780-795. https://doi.org/10.1016/j.neuro n.2016.09.050

34. Mizutani T, Aki M, Shiozawa R, Unakami M, Nozawa T, Yajima K, Tanabe H, Hara M (1990) Development of ophthalmoplegia in amyotrophic lateral sclerosis during long-term use of respirators. J Neurol Sci 99:311-319

35. Neumann M, Sampathu DM, Kwong LK, Truax AC, Micsenyi MC, Chou TT, Bruce J, Schuck T, Grossman M, Clark CM, McCluskey LF, Miller BL, Masliah E, Mackenzie IR, Feldman H, Feiden W, Kretzschmar HA, Trojanowski JQ, Lee VM (2006) Ubiquitinated TDP-43 in frontotemporal lobar degeneration and amyotrophic lateral sclerosis. Science 314:130-133. https://doi. org/10.1126/science. 1134108

36. Philips T, De Muynck L, Thu HN, Weynants B, Vanacker P, Dhondt J, Sleegers K, Schelhaas HJ, Verbeek M, Vandenberghe R, Sciot R, Van Broeckhoven C, Lambrechts D, Van Leuven F, Van Den Bosch L, Robberecht W, Van Damme P (2010) Microglial upregulation of progranulin as a marker of motor neuron degeneration. J Neuropathol Exp Neurol 69:1191-1200. https:// doi.org/10.1097/NEN.0b013e3181fc9aea 
37. Polymenidou M, Lagier-Tourenne C, Hutt KR, Huelga SC, Moran J, Liang TY, Ling SC, Sun E, Wancewicz E, Mazur C, Kordasiewicz H, Sedaghat Y, Donohue JP, Shiue L, Bennett CF, Yeo GW, Cleveland DW (2011) Long pre-mRNA depletion and RNA missplicing contribute to neuronal vulnerability from loss of TDP-43. Nat Neurosci 14:459-468. https://doi.org/10.1038/nn.2779

38. Prudencio M, Belzil VV, Batra R, Ross CA, Gendron TF, Pregent LJ, Murray ME, Overstreet KK, Piazza-Johnston AE, Desaro P, Bieniek KF, DeTure M, Lee WC, Biendarra SM, Davis MD, Baker MC, Perkerson RB, van Blitterswijk M, Stetler CT, Rademakers R, Link CD, Dickson DW, Boylan KB, Li H, Petrucelli L (2015) Distinct brain transcriptome profiles in C9orf72-associated and sporadic ALS. Nat Neurosci 18:1175-1182. https://doi. org/10.1038/nn.4065

39. Rabin SJ, Kim JM, Baughn M, Libby RT, Kim YJ, Fan Y, Libby RT, La Spada A, Stone B, Ravits J (2010) Sporadic ALS has compartment-specific aberrant exon splicing and altered cellmatrix adhesion biology. Hum Mol Genet 19:313-328. https:// doi.org/10.1093/hmg/ddp498

40. Ravits J, Laurie P, Fan Y, Moore DH (2007) Implications of ALS focality: rostral-caudal distribution of lower motor neuron loss postmortem. Neurology 68:1576-1582. https://doi. org/10.1212/01.wnl.0000261045.57095.56

41. Ravits J, Paul P, Jorg C (2007) Focality of upper and lower motor neuron degeneration at the clinical onset of ALS. Neurology 68:1571-1575. https://doi.org/10.1212/01.wnl.0000260965.20021 .47

42. Renton AE, Chio A, Traynor BJ (2014) State of play in amyotrophic lateral sclerosis genetics. Nat Neurosci 17:17-23. https:// doi.org/10.1038/nn.3584

43. Rodrigues MC, Hernandez-Ontiveros DG, Louis MK, Willing AE, Borlongan CV, Sanberg PR, Voltarelli JC, Garbuzova-Davis S (2012) Neurovascular aspects of amyotrophic lateral sclerosis. Int Rev Neurobiol 102:91-106. https://doi.org/10.1016/B978-012-386986-9.00004-1
44. Salado IG, Redondo M, Bello ML, Perez C, Liachko NF, Kraemer BC, Miguel L, Lecourtois M, Gil C, Martinez A, Perez DI (2014) Protein kinase CK-1 inhibitors as new potential drugs for amyotrophic lateral sclerosis. J Med Chem 57:2755-2772. https://doi. org/10.1021/jm500065f

45. Scelsa SN, Yakubov B, Salzman SH (2002) Dyspnea-fasciculation syndrome: early respiratory failure in ALS with minimal motor signs. Amyotroph Lateral Scler Other Motor Neuron Disord 3:239-243

46. Sta M, Sylva-Steenland RM, Casula M, de Jong JM, Troost D, Aronica E, Baas F (2011) Innate and adaptive immunity in amyotrophic lateral sclerosis: evidence of complement activation. Neurobiol Dis 42:211-220. https://doi.org/10.1016/j.nbd.2011.01.002

47. Taylor JP, Brown RH Jr, Cleveland DW (2016) Decoding ALS: from genes to mechanism. Nature 539:197-206. https://doi. org/10.1038/nature20413

48. Turner MR, Cagnin A, Turkheimer FE, Miller CC, Shaw CE, Brooks DJ, Leigh PN, Banati RB (2004) Evidence of widespread cerebral microglial activation in amyotrophic lateral sclerosis: an [11C](R)-PK11195 positron emission tomography study. Neurobiol Dis 15:601-609. https://doi.org/10.1016/j.nbd.2003.12.012

49. Van Nostrand EL, Pratt GA, Shishkin AA, Gelboin-Burkhart C, Fang MY, Sundararaman B, Blue SM, Nguyen TB, Surka C, Elkins K, Stanton R, Rigo F, Guttman M, Yeo GW (2016) Robust transcriptome-wide discovery of RNA-binding protein binding sites with enhanced CLIP (eCLIP). Nat Methods 13:508-514. https://doi.org/10.1038/nmeth.3810

50. Walker AK, Spiller KJ, Ge G, Zheng A, Xu Y, Zhou M, Tripathy K, Kwong LK, Trojanowski JQ, Lee VM (2015) Functional recovery in new mouse models of ALS/FTLD after clearance of pathological cytoplasmic TDP-43. Acta Neuropathol 130:643660. https://doi.org/10.1007/s00401-015-1460-x 